\title{
Comparison and Process Optimisation of PLGA, Chitosan and Silica Nanoparticles for Potential Oral Vaccine Delivery.
}

\author{
Muhammad K. Amin ${ }^{1}$, Joshua S. Boateng ${ }^{1 *}$.
}

${ }^{1}$ School of Science, Faculty of Engineering and Science, University of

Greenwich, Medway, Kent, ME4 4TB

*Corresponding Author

Email: J.S.Boateng@gre.ac.uk; joshboat40@gmail.com

Tel: +44 (0) 7525630830

Fax: +44 (0) 2083319805 


\begin{abstract}
:
Aims: The study compared performance of nanoparticles from synthetic organic, natural organic and inorganic materials as vaccine delivery platforms.

Methods: Various formulation (concentration, polymer/silica: surfactant ratio, solvent) and process parameters (homogenization speed and time, ultra-sonication) affecting functional performance characteristics of poly(lactic-co-glycolic acid) (PLGA), chitosan and silica based nanoparticles containing bovine serum albumin were investigated. Nanoparticles were characterised using dynamic light scattering, X-ray diffraction, scanning/transmission electron microscopy, Fourier transform infrared spectroscopy and in vitro protein release.

Results: Critical formulation parameters were surfactant concentration (PLGA, silica) and polymer concentration (chitosan). Optimised nanoparticles were spherical in shape with narrow size distribution and size ranges of 100-300 $\mathrm{nm}$ (blank) and 150-400 nm (protein loaded). Protein encapsulation efficiency was $26-75 \%$ and released within 48 hours in a sustained manner.

Conclusion: Critical formulation and process parameters affected size of PLGA, chitosan and silica nanoparticles and protein encapsulation, whilst silica produced the smallest and most stable nanoparticles.
\end{abstract}

Keywords: Bovine serum albumin, Chitosan, Nanoparticles, Oral Vaccination, poly(lactic-coglycolic acid), Process Optimization, Protein Delivery, Silica 


\section{Introduction}

In the last decade, advancements in nano-engineering and material science, have made nanoparticles very attractive and have been widely investigated in the field of drug delivery and nanomedicine[1] [2]. Nano carriers might help to deliver therapeutic proteins via the oral route due to two main characteristics: (i) as a protective shield against the harsh host biological environment and (ii) as a carrier to cross the mucosal epithelia (membrane) in the gastrointestinal tract (GIT) [3]. Recently, polymeric nanoparticles have received increased attention in oral protein delivery due to their stability, biodegradability, and biocompatibility in biological systems and ease of surface modification to suit various applications [4] as well as ability to protect therapeutic proteins against enzymatic (e.g. nucleases and proteases) degradation [5]. Protein encapsulation in polymeric nanoparticles is a very challenging but promising area in protein delivery research [6] [7], therefore fundamental and systematic formulation development and optimization of selected delivery systems to reach the intended site of action is an important endeavour.

Poly(D,L-lactide-co-glycolide) or poly(lactic-co-glycolic acid) (PLGA) is one of the most widely used polymers for formulating nanoparticle carriers in protein/peptide oral delivery [8]. The Food and Drugs Administration (FDA) and European Medicine Agency (EMA) approved poly-lacticco-glycolic acid (PLGA) for various drug delivery applications such as blends, films, matrices, microspheres, pellets and nanoparticles [9]. PLGA nanoparticles have been proven to be capable of encapsulating and protecting protein molecules against harsh biological environments, allow sustained release and improve their therapeutic efficacy [10]. Since 1990, chitosan has been studied as drug delivery material in the form of film forming matrices for controlled release of drugs with the ability to temporally open intracellular epithelial tight junctions and facilitate uptake of hydrophilic drugs [11]. In the mid to late 1990s, a new self-assembling amphiphilic form of chitosan was introduced for formulating chitosan nanoparticles [12]. To determine and characterize the functional properties of chitosan, molecular weight, polydispersity and degree of deacetylation are important factors which greatly affect the particle size, particle formation and aggregation [12]. Various researchers have reported the formulation of chitosan nanoparticles as protein delivery carrier to protect entrapped proteins from enzymatic degradation in biological systems [13][14][15]. One of the major advantages of chitosan is its ability to form positively charged particles under mild agitation conditions without the need for harmful organic solvents and facilitate the adsorption or encapsulation of therapeutic proteins and antigens or form polyplexes by electrostatic interaction with negatively charged nucleotides [16]. Mesoporous silica nanoparticles (MSNs) are a class of molecules that have attracted attention in the delivery of small molecules, due to their many desirable properties. They are easily synthesised with open pore structure, and the size of the pores and particle surface can be controlled during synthesis [17]. Furthermore, the silanol-containing surface of silica nanoparticles can be easily functionalized with bio-recognition entities which allows targeting of specific cells or receptors in the body [18]. Due to their mesoporous structure, silica nanoparticles can easily encapsulate protein molecules and protect 
them from harsh biological environments in the body, thereby increasing the efficiency of protein delivery, reducing renal clearance and ultimately increasing duration of action [19]. Since silica nanoparticles are much smaller than eukaryotic cells, they allow the use of protein transport in the cytosol via an endocytosis pathway and subsequent endosomal escape [20].

The aim of this study was the formulation development and subsequent comparison of nanocarriers obtained from three different types of materials (i) synthetic polymer (PLGA), (ii) biomaterial organic polymer (chitosan) and (iii) inorganic (silica). In addition we have investigated bovine serum albumin (BSA) encapsulation as a model protein with special emphasis on important physicochemical properties and processing parameters to achieve optimised characteristics for both polymeric and inorganic nanoparticles that will be suitable for potential mucosal vaccination. Though various studies have investigated the use of individual synthetic, natural or inorganic based nanoparticles for protein delivery, no study has systematically compared nanoparticles obtained from different sources for protein encapsulation in a single study. Therefore the novelty of the study lies in the fact that, to the best of our knowledge, this is the first study that systematically compares nanoparticles obtained from synthetic organic (PLGA), natural organic (chitosan), and inorganic starting materials and simultaneously investigating the effect of process and formulation variables on the performance characteristics of the resulting nanoparticles for potential protein delivery.

\section{Materials and Methods}

\subsection{Materials}

Poly(D,L-lactide-co-glycolide) lactide: glycolide (50:50), MW 30,000-60,000 (Lot, P2191), chitosan (medium molecular weight, 75-85\% deacetylated, lot no 448877), poly (vinyl alcohol) MW 89000$98000,99+\%$ hydrolysed tetraethyl orthosilicate, MW 208.33, cetyltrimethylammonium bromide, MW 62,000, Tween 80 average MW 1310, Pluronic ${ }^{\circledR}$ F-127 CAS N0: 9003-11-6, sodium tripolyphosphate, and bovine serum albumin were purchased from Sigma-Aldrich, (Gillingham, UK). Glacial acetic acid, calcium chloride $\left(\mathrm{CaCl}_{2}\right)$, Coomassie brilliant blue, disodium hydrogen phosphate $\left(\mathrm{Na}_{2} \mathrm{HPO}_{4}\right)$, hydrochloric acid $(\mathrm{HCl})$, monobasic potassium dihydrogen phosphate $\left(\mathrm{KH}_{2} \mathrm{PO}_{4}\right)$, sodium acetate, sodium azide $\left(\mathrm{NaN}_{3}\right)$, sodium bicarbonate $\left(\mathrm{NaHCO}_{3}\right)$, sodium chloride $(\mathrm{NaCl})$, sodium chloride $(\mathrm{NaCl})$, sodium dihydrogen phosphate, $\mathrm{NaH}_{2} \mathrm{PO}_{4}$, sodium hydroxide $(\mathrm{NaOH})$, and $\mathrm{D}(+)$ trehalose were purchased from (Thermo Fisher Scientific, Loughborough, UK).

\subsection{Formulation development}

\subsubsection{Preparation of PLGA nanoparticles}

PLGA nanoparticles were prepared by solvent emulsification evaporation and double emulsion (w/o/w) technique as previously reported [21] but with some modifications. Briefly, 100mg PLGA was dissolved in $5 \mathrm{ml}$ dichloromethane (DCM) and vortexed until a clear solution was obtained, $0.5 \mathrm{ml}$ phosphate buffered saline ( $\mathrm{pH} 7.4$ ) was added and sonicated for $30 \mathrm{~s}$ at amplitude of $70 \%$ to obtain a 
primary emulsion. Different amounts of polyvinyl alcohol (PVA) were dissolved in 100ml of deionised water and the temperature increased up to $60^{\circ} \mathrm{C}$ with stirring at 300rpm for $30 \mathrm{~min}$ until a clear solution $(1-4 \% \mathrm{w} / \mathrm{v})$ was obtained and then cooled to room temperature. The primary emulsion was added dropwise into $50 \mathrm{ml}$ of the $1 \% \mathrm{w} / \mathrm{v}$ PVA and sonicated as described above and this was repeated for the other PVA $(2 \%, 3 \%$ and $4 \% \mathrm{w} / \mathrm{v})$ solutions respectively. The DCM was then evaporated by stirring continuously on a magnetic stirrer at 300rpm for $6 \mathrm{hrs}$. Homogeneous nanoparticles were obtained with the help of a homogenizer at different speeds $(1000-10,000 \mathrm{rpm})$ and duration $(5-25 \mathrm{~min})$ of stirring. The procedure was repeated for two other surfactants (Tween 80 - TW and Pluronic F127 - PL) in place of PVA. The final formulated nanoparticle emulsion was centrifuged at $15000 \mathrm{rpm}$ for $30 \mathrm{~min}$, pellet collected, washed 3 times with deionized water, freeze dried and characterized as a blank nanoparticle. To obtain protein loaded nanoparticles, freeze dried blank nanoparticles were dispersed in deionized water, $20 \mathrm{mg} / \mathrm{ml}$ of bovine serum albumin (BSA) solution was added dropwise into $80 \mathrm{ml}$ of dispersed lyophilized PLGA nanoparticle suspension to achieve a final volume of $100 \mathrm{ml}$ and sonicated for $60 \mathrm{~s}$ whilst placed on ice to avoid heat generation in the solution. Finally, protein loaded nanoparticles were centrifuged at $15000 \mathrm{rpm}$ for $30 \mathrm{~min}$ and both supernatant and pellet were collected to analyse the amount of protein encapsulation in the nanoparticle and free protein in the supernatant.

\subsubsection{Preparation of chitosan nanoparticles}

Chitosan nanoparticles were prepared by following inotropic gelation method [22] using medium molecular weight chitosan. Chitosan was dissolved in an aqueous solution of acetic acid $(1 \% \mathrm{v} / \mathrm{v})$ at different chitosan concentrations (Figure S1, Supplementary data) and allowed to fully dissolve (60 $\mathrm{min}$ ). Then $1 \mathrm{mg} / \mathrm{ml}$ sodium tripolyphosphate (TPP) solution was added dropwise to the chitosan solution with magnetic stirring (600rpm). The blank nanoparticles were formed during stirring at room temperature of chitosan-TPP suspension for $60 \mathrm{~min}$ and sample collected as blank nanoparticles for characterisation. Protein loaded chitosan-TPP nanoparticle suspensions were prepared by simply adding the BSA solution $(0.5 \mathrm{mg} / \mathrm{ml})$ without adding any organic solvents or high energy (e.g. sonication) sources. The BSA loaded chitosan-TPP nanoparticle suspension was gently stirred for $4 \mathrm{hrs}$ at room temperature and the resulting particles collected for further analysis. The incorporation technique described were used to prepare a total of 4 different protein loaded nanoparticle formulations using the above experimental variables (i.e. chitosan-TPP ratio) described for the blank equivalents.

\subsubsection{Preparation of silica nanoparticles}

Silica nanoparticles were prepared by sol-gel synthesis process with slight modification [23]. $2 \mathrm{ml}$ of tetraethylorthosilicate (TEOS) and $2 \mathrm{ml} \mathrm{HCl}(0.0035 \mathrm{M})$ were mixed together and stirred for $30 \mathrm{~min}$ under ambient temperature, sonicated for $30 \mathrm{~s}$ and then stirred again for $2 \mathrm{hrs}$ until it became transparent. After that, pre-determined amounts $(0.1 \%-1.0 \%)$ of cetyltrimethylammonium (CTAB) surfactant solutions were added separately. The TEOS and surfactant solutions were stirred at $80^{\circ} \mathrm{C}$ for $2 \mathrm{hrs,} \mathrm{till}$ 
a solid white precipitate was obtained. The temperature was reduced slowly and the white precipitate centrifuged $(15,000 \times \mathrm{rpm})$ to separate out the solid blank nanoparticles. The white product was washed four times, (once with ethanol and three times with Millipore deionized water). The ethanol washing was done to remove excess amounts of surfactants. After centrifuging, the blank silica nanoparticles were dispersed in $80 \mathrm{ml}$ deionized water and stirred for $1 \mathrm{hr} .20 \mathrm{ml}$ of BSA $(5 \mathrm{mg} / \mathrm{ml})$ solution was added slowly into the resulting blank silica nanoparticles suspension and stirred cold at room temperature for $24 \mathrm{hrs}$ to prepare protein loaded nanoparticles. The BSA loaded mixture was centrifuged at $15000 \times \mathrm{rpm}$ (Hitachi) and supernatant separated from pellet and free protein concentration in the supernatant analysed using Bradford protein assay.

\subsection{Protein encapsulation and loading studies}

After overnight incubation, protein-loaded PLGA, chitosan and silica nanoparticles were collected by centrifugation (15000 rpm, $30 \mathrm{~min}$ ) and separated from the non-encapsulated (free) BSA. BSA concentration was calculated from Bradford assay using the equation of a calibration curve. This was achieved by measuring the fluorescence intensity and absorbance of BSA solutions loaded in 96-well plates with the help of a plate reader (Thermofisher multiskan, photometer). The standard curves were based on the absorbance of protein within the concentration range of $0.1 \mathrm{mg} / \mathrm{ml}$ to $3 \mathrm{mg} / \mathrm{ml}$. The calibration curves were based on the absorbance at 620 and 540, respectively, as a function of concentration. The EE \% and loading capacity $(\mathrm{mg} / \mathrm{g})$ were calculated as shown in equations $1 \& 2$.

$$
\begin{aligned}
& \mathbf{E E}(\%)=\frac{\text { total amount of BSA-free BSA in supernatent }}{\text { total amount of BSA }} \times 100 \\
& \mathbf{L C}(\%)=\frac{\text { total amount of BSA-free BSA in supernatent }}{\text { Dried nanoparticles weight }} \times 100
\end{aligned}
$$

\subsubsection{Limit of detection (LOD) and limit of quantitation (LOQ)}

LOD and LOQ were calculated from the data obtained from the linearity plot. For each of the three replicate determinations of same concentration, standard deviation (SD) of the responses was calculated.

Limit of detection (LOD) and limit of quantitation (LOQ) were calculated by using equations 3 and 4:

$$
\begin{gathered}
\text { LOD }=\frac{3.3 \alpha}{\mathrm{S}} \\
\text { LOQ }=\frac{3 \alpha}{\mathrm{S}} \ldots .
\end{gathered}
$$

Where $\alpha=$ Standard deviation of the response; $\mathrm{S}=$ Slope of the calibration curve.

Range of $100-2000 \mu \mathrm{g} / \mathrm{ml}$, linear regression (LR) $\mathrm{Y}=0.4625 \mathrm{x}+0.7656, \mathrm{R}^{2}=0.9978, \mathrm{LOD}=0.2 \mu \mathrm{g} / \mathrm{ml}$ and $\mathrm{LOQ}=0.87 \mu \mathrm{g} / \mathrm{ml}$. 


\subsection{Formulation process optimisation}

Different parameters that affect the functional properties of the nanoparticles were investigated as part of formulation development and optimization process.

\subsubsection{Concentration of polymer/silica and surfactant ratio}

To determine the optimum surfactant concentration in aqueous phase during the PLGA nanoparticle preparation, three different types of surfactants (polyvinyl alcohol-PVA, Pluronic-PL and Tween 80TW) were used and different surfactant concentrations $(0.1 \%, 0.5 \%, 1 \%$ and $1.5 \% \mathrm{w} / \mathrm{v})$ were also prepared and their effect on nanoparticle size, polydispersity index (PDI) and zeta potential measured. To determine the chitosan and TPP ratio, different concentrations of chitosan $(0.1 \%, 0.2 \%, 0.3 \%$ and $0.4 \%$ ) were formulated at fixed amount of TPP $1 \mathrm{mg} / \mathrm{ml}$. For silica nanoparticles, CTAB was employed at different concentrations from $(0.1 \%-1.0 \%)$ with fixed amount of TEOS $2 \mathrm{ml}(\mathrm{v} / \mathrm{v})$.

\subsubsection{Effect of homogenizer speed and sonication time.}

Different formulation batches were prepared at various homogenization speeds $(1000,3000,5000,7000$ and $10000 \mathrm{rpm})$ respectively and their sizes, PDI and zeta potential were measured. Sonication was performed at different amplitudes (30\%, 40\%, and 50\%) and over different time periods (1 - $12 \mathrm{~min})$ and the effect on particle size, PDI and zeta potential evaluated.

\subsection{Physicochemical characterization of nanoparticle properties}

\subsubsection{Particle size evaluation using dynamic laser scattering (DLS)}

Particle size, polydispersity index and zeta potential were measured using a Zetasizer Nano-ZS90 (Malvern Instruments, UK). Disposable sizing cuvettes were used for size and PDI analysis while zeta potential were measured using a reusable folded capillary Zeta Cell, Malvern Model: DTS1070. The samples was measured in double distilled water and adjusted to a conductivity of $50 \mathrm{IS} / \mathrm{cm}$ with sodium chloride solution $(0.9 \% \mathrm{w} / \mathrm{v})$. The $\mathrm{pH}$ was in the range of 5.5-7.5 and the applied field strength was 20 $\mathrm{V} / \mathrm{cm}$. The measurements were made at a position of $4.65 \mathrm{~mm}$ from the cuvette wall with an automatic attenuator and at a controlled temperature of $20^{\circ} \mathrm{C}$. All the measurements were performed in triplicate $(n=3)$ and data were presented as mean \pm SD.

\subsubsection{Morphology by scanning and transmission electron microscopy}

The morphology of the PLGA, chitosan and silica nanoparticles was examined by scanning electron microscopy (SEM) (Hitachi SU8030) at operating voltage of $1 \mathrm{kV}$. One drop of freshly prepared particle suspensions was deposited on sample stub, allowed to dry in air and the air dried sample was coated with chromium for $3 \mathrm{~min}$. Low voltage $(1 \mathrm{kV})$ was set for observing PLGA nanoparticles to avoid heating by strong electron stream which caused particle deformation and decomposition. Further a high resolution transmission electron microscope (TEM) was used to examine morphological characteristic of nanoparticles. One drop of sample was placed on 300 mesh copper grid, allowed to sit for $10 \mathrm{~min}$ until air dried and excess liquid removed using filter paper before observation on the TEM machine. 
For scanning transmission electron microscopy (STEM) analysis, $30 \mathrm{kV}$ high-resolution instrument was used to examine morphological characteristic of nanoparticles.

\subsubsection{Attenuated total reflectance-Fourier transform infrared (ATR-FTIR) spectroscopy}

The infrared spectra were obtained for both pure starting materials and the various nanoparticles at $20^{\circ} \mathrm{C}$ for wavenumbers ranging from $4000-450 \mathrm{~cm}^{-1}$. ATR-FTIR analyses were performed in absorption mode using a Perkin Elmer FT-IR spectrophotometer. Different peaks in the IR spectrum were interpreted for different functional groups in the formulations.

\subsubsection{X-ray diffraction (XRD)}

$\mathrm{X}$-ray diffraction was performed to investigate crystalline or amorphous nature of the formulated nanoparticles. The diffractograms were recorded using a Bruker AXS D8 Advance instrument with $\mathrm{Cu}-$ $\mathrm{ka}$ line as a source of radiation and operated at a voltage of $40 \mathrm{keV}$ and current of $35 \mathrm{~mA}$. Freeze dried formulated samples and pure starting materials were measured in a $2 \theta$ range between $3^{\circ}$ and $80^{\circ}$ for 10 $\min$ at a step size of $5 \mathrm{~s}$.

\subsubsection{In-vitro protein release study}

To determine the amount of protein released from PLGA, chitosan and silica nanoparticles, the Bradford reagent (protein-specific dye, Coomassie brilliant blue) was used and analysed in a 96 -well polystyrene plate. Freeze dried protein loaded nanoparticles $(5 \mathrm{mg} \mid)$ were dispersed in $0.01 \%$ sodium azide containing $2 \mathrm{ml}$ of PBS ( $\mathrm{pH} \mathrm{7.4)}$ ) and incubated at $37^{\circ} \mathrm{C}$ in bath shaker with gentle stirring. At predetermined time intervals over $48 \mathrm{hrs}, 0.1 \mathrm{ml}$ aliquots of dissolution medium were collected and replaced with same amount of fresh PBS. The samples $(n=3)$ were analysed with absorbance plate reader at wavelength of $595 \mathrm{~nm}$ and Bradford reagent standards were used according to manufacturer's instruction kit.

\section{6 Statistical analysis}

All the quantitative data from DLS and protein analyses were analysed by one-way ANOVA using GraphPad Prism software (version 5.02). The level of significance was set at $p<0.05$.

\section{Results and discussion:}

\subsection{Effect of concentration of polymer, silica and surfactant on particle properties.}

The various formulation and process parameters (nanoparticle size, zeta potential, PDI, morphology and emulsion composition) were considered in order to select the optimum formulation(s) for encapsulating proteins as well as comparing the performance characteristics of optimised PLGA, chitosan and silica nanoparticles. Generally, the optimised PLGA, chitosan and silica nanoparticles had size below $300 \mathrm{~nm}$ as was originally intended (Figure 1). The results (Figure S1) showed that the amount of stabilizing agent and polymer affected the particle size, PDI and zeta potential of PLGA nanoparticles. The DLS results for PLGA-PVA formulations showed that when PVA amount increased, size decreased, and zeta potential increased which can be attributed to the increased ability of the surfactant to reduce nanoparticle surface tension and promote the break-up of larger particles during 
homogenization. It is well established that nanoparticle surface charge also plays a vital role to interact with target cells upon administration and the PLGA nanoparticles showed a negative surface charge due to the anionic properties of PLGA and the cationic surfactant [21].

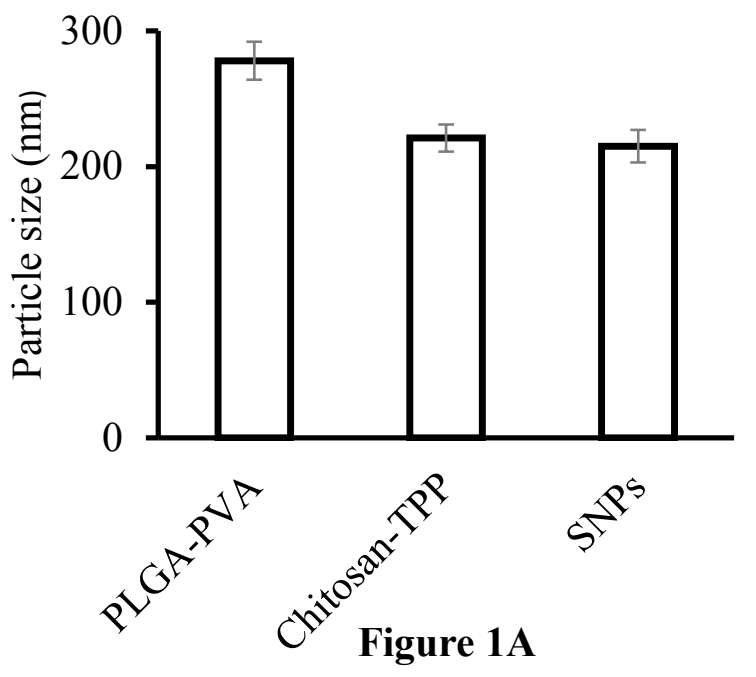

PLGA-PVA

Chitosan-TPP
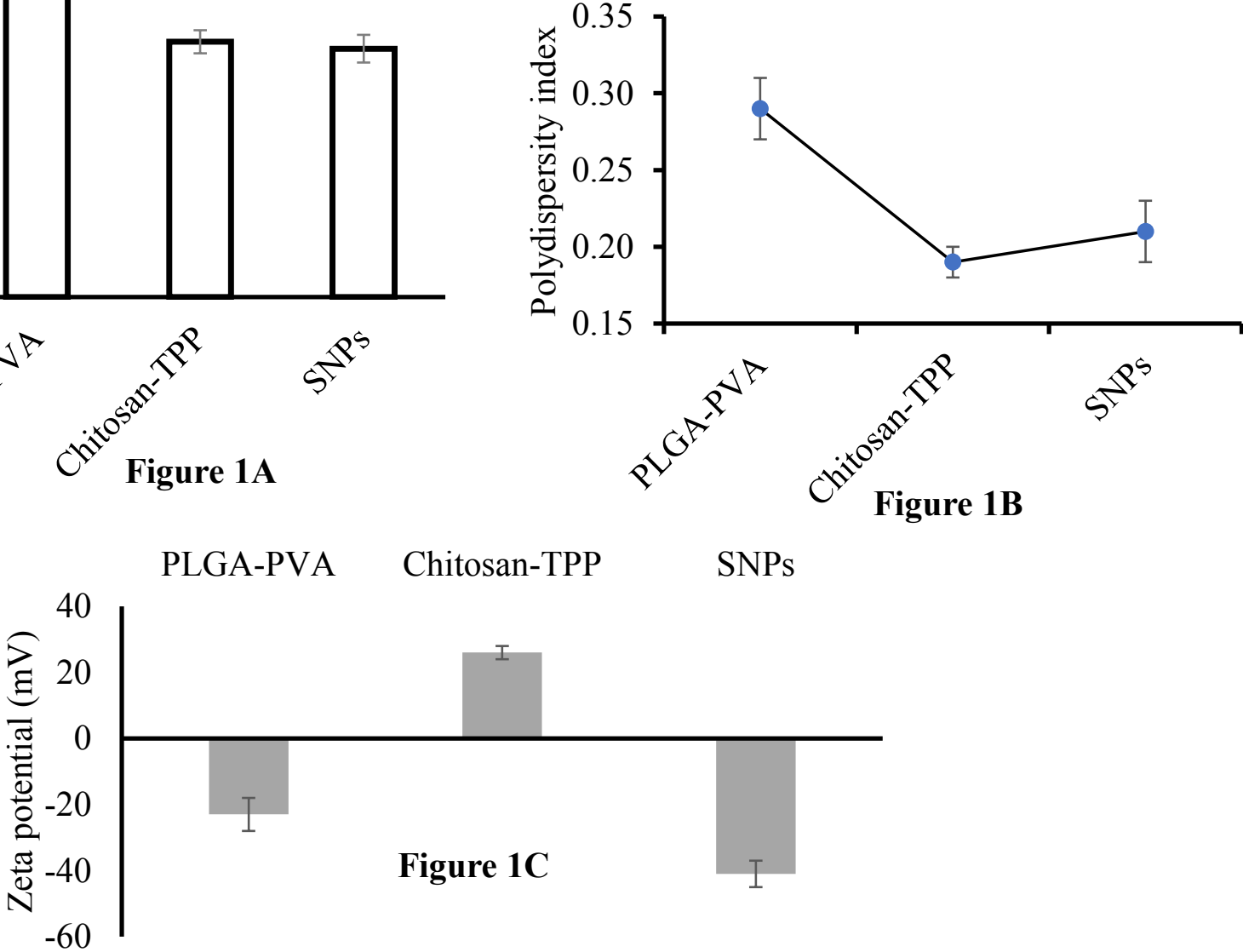

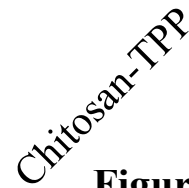

Figure 1B

Figure 1. Figure 1 DLS results showing (A) particle size, (B) PDI and (C) zeta potential of selected optimised nanoparticles prepared with poly(lactic-co-glycolic acid)-polyvinyl alcohol (PLGA-PVA), chitosan-sodium tripolyphosphate (TPP) and silica (SNPs) $(n=3)$

Higher concentrations of surfactant resulted in better stabilized nanoparticles with desirable size and surface charge properties was not affected. It was also observed that, generally, the PDI of the PLGA nanoparticles was low which indicates narrow size distribution, and this was also influenced by the surfactant ratio. For PLGA and PL based formulations, at $0.1 \%$ to $1.5 \%$ surfactant content, the PDI decreased $(p<0.05)$ from $0.52 \pm 0.2$, to $0.12 \pm 0.2$ respectively. Increasing the amount of TW from $0.1 \%$ to $1.5 \%$ also increased $(p<0.05)$ the particle diameter from $266 \pm 6 \mathrm{~nm}$ to $309 \pm 10 \mathrm{~nm}$. In this case two factors were involved: a) when organic phase (PLGA/DCM) was added dropwise into the aqueous phase (surfactant solution), the solution became more viscous and reduced the net shear stress with possibility to make bigger droplets; b) when viscosity increased in surfactant solution, there was a decrease in the dispersion of aqueous phase resulting in increased particle diameter. PLGA 
nanoparticles prepared from emulsions containing $1 \%$ and $1.5 \%$ concentrations of PVA (surfactant) were selected for protein encapsulation.

Nanoparticles from chitosan were successfully formed through ionic gelation of chitosan with TPP acting as the cross-linking moiety. The process yielded homogenously dispersed nanoparticles $>100 \mathrm{~nm}$ in size, with low PDI and generally high zeta potential values via a simple and easily reproducible method. The chitosan-TPP nano systems demonstrate a wide range of attractive features which render them promising as carriers of oral protein delivery [12, 24]. For chitosan nanoparticles, the particle size was linearly increased $(p<0.05$ ) from $221 \mathrm{~nm}$ to $346 \mathrm{~nm}$ by increasing chitosan concentration from $0.1 \%$ to $0.4 \%$, possibly due to presence of intermolecular hydrogen bonding (-OH) and intermolecular electrostatic repulsion with $-\mathrm{NH}_{3}{ }^{+}$which exist on the chitosan surface [25].

When chitosan concentration was increased from $0.1 \%$ to $0.4 \%$ (Figure S3, supplementary data) there was slight increase $(p>0.05)$ of zeta potential from +26 to $+29 \mathrm{mV}$ except $0.2 \%$ formulation. This is because, the positive surface charge on chitosan -TPP nanoparticle does not interact with TPP ions due to the presence of residual amine groups [22]. The slight decrease of zeta potential value +26 to $+24 \mathrm{mV}$ on $0.2 \%$ could be caused by the $\mathrm{H}^{+}$liberated from the acetic acid $\left(\mathrm{CH}_{3} \mathrm{C}^{-} 0^{-} \mathrm{H}^{+}\right)$which had been used up to protonate the $\mathrm{NH}_{2}$ groups on the backbone of chitosan to allow the polymer to fully dissolve during formulation [26]. Similarly, when chitosan concentration increased from $0.1 \%$ to $0.4 \%$, the PDI also increased $(p<0.05)$ from 0.19 to 0.27 due to the fact that higher concentration tends to increase the agglomeration of particles which in turn prompt an increased polydispersity of the particles (Figure S2). Overall, nanoparticles obtained from chitosan: TPP ratio of 1:1 with $0.1 \%$ total chitosan, showed good particle size, PDI and zeta potential values.

From the DLS measurement it was observed that, silica nanoparticles were not formed effectively at $\mathrm{CTAB}$ concentrations from $0.1 \%$ to $0.5 \%$. The PDI values of these formulations (silica 1 to silica 5) were high (0.6 to 0.4 ) due to the absence of monodispersed particles and dispersion solution not being homogenous. The particles size ranged from $1000 \mathrm{~nm}$ to $3000 \mathrm{~nm}$ for silica 1 to silica 5 samples (FigureS3, supplementary data), while zeta potential values indicated low stability of particles in solution. At higher concentration $(0.6 \%)$ of $\mathrm{CTAB}$ nano sized particle formation was observed with mean particles size in the range of 230 to $650 \mathrm{~nm}$. Monodisperse particles with uniform size of $132 \pm 2$ $\mathrm{nm}$ was observed for the silica 9 sample prepared with CTAB concentration of $0.9 \%$ and was chosen as optimum concentration for protein loading. However, the formulations obtained from CTAB concentrations of $0.9 \%$ and $1 \%$, showed larger particle sizes under the same experimental conditions due to particle agglomeration.

\subsection{Effect of homogenizer speed and duration on nanoparticle size, PDI and zeta potential.}

In addition to the concentration of starting materials, homogenizer speed and total time of mixing also affected the size, PDI and zeta potential of all the three types (PLGA, chitosan and silica) of nanoparticles. In Figure 2 it was observed that the homogenizer speed had a remarkable effect on PLGA 
nanoparticle size and dispersion, with particle size decreasing and PDI increasing as the homogenizer speed increased. At low homogenizer speed (1000rpm) over $10 \mathrm{~min}$, the size was $2547 \pm 152 \mathrm{~nm}$, PDI $0.93 \pm 0.4$ and zeta potential $-8.40 \pm 3.8 \mathrm{mV}$. However, increasing the homogenizer speed gradually to $10000 \mathrm{rpm}$ at fixed time of stirring (P5) showed a significant $(p<0.05))$ reduction in particle size to $290 \pm 7$, PDI to $0.10 \pm 0.1$ while zeta potential increased to $-12 \pm 6.8$. Further, Figure S4 and S5 (supplementary data) show effects of homogenizer speeds below 10,000 and effect of homogenizer speed at fixed time respectively on properties of PLGA nanoparticles. The faster the suspension was stirred, the more stable the particles appeared based on the zeta potential. Increasing high shear stress leads to the formation of smaller particles resulting in repulsive forces between the similarly charged particles, preventing their aggregation and therefore increase in their stability. For a fixed stirring speed, the particle size decreased and zeta potential increased with increasing homogenizer stirring time. Furthermore, the data also shows that the optimum stirring speed and time were $10000 \mathrm{rpm}$ and $25 \mathrm{~min}$ respectively because above these values, the suspension begins to foam and generated a lot of air bubbles and with possibility for particle aggregation and reduction in zeta potential. 

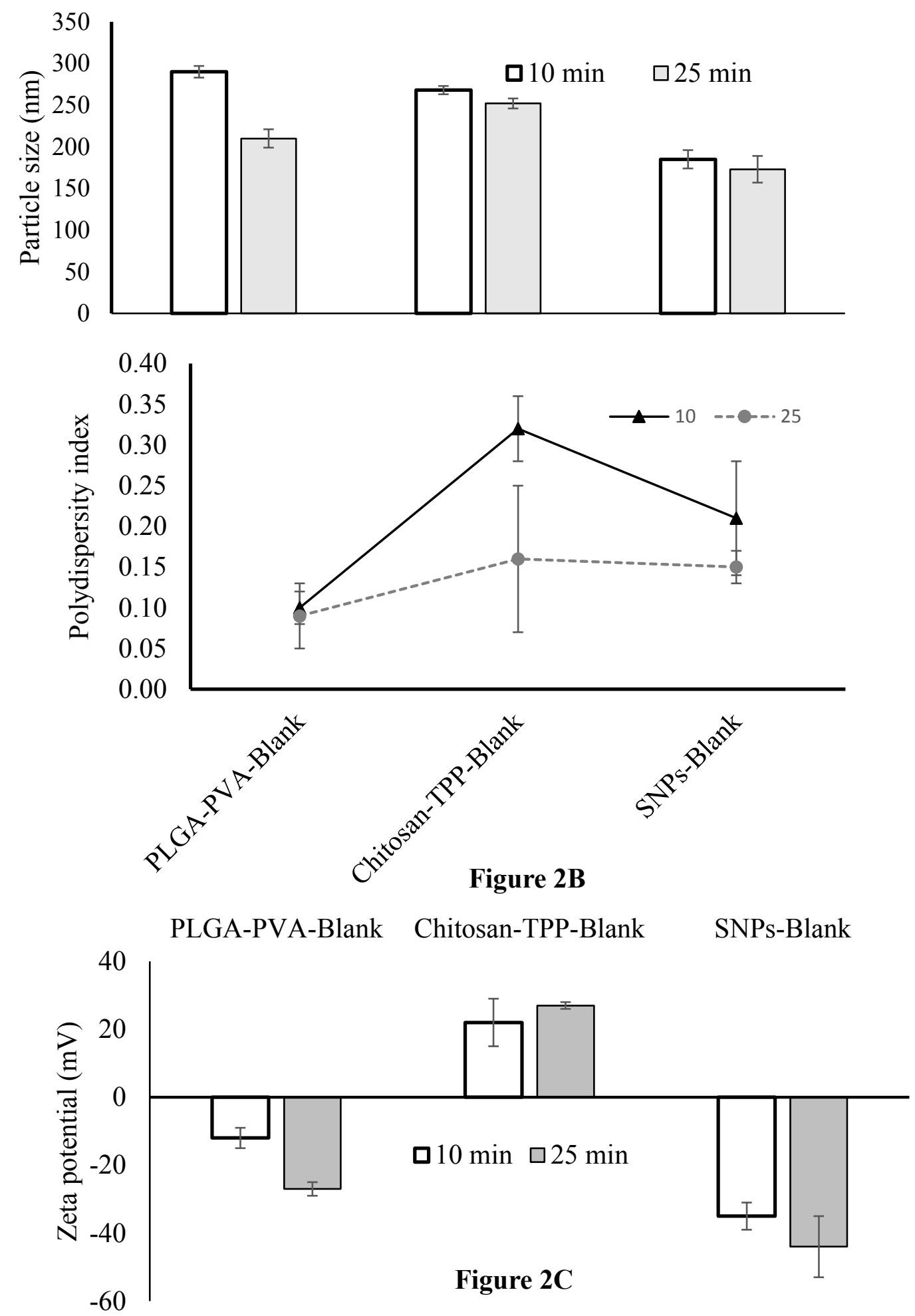

Figure 2: Investigation of effect of homogenizer time at fixed stirring speed $(10,000 \mathrm{rpm})$ for blank poly(lactic-co-glycolic acid)-polyvinyl alcohol (PLGA-PVA), chitosan- sodium tripolyphosphate (TPP) and silica (SNPs) nanoparticles $(n=3)$. 
Different homogenization speeds were employed in a representative chitosan nanoparticle formulation $(0.1 \%)$ prepared from chitosan $100 \mathrm{mg}$ and $0.1 \%$ TPP. Narrow and small size range of nanoparticles diameter was obtained at 7000rpm in $10 \mathrm{~min}$ which was selected as homogenizer speed for further investigation. Homogeniser speeds beyond 7000 caused the nanoparticle formulations to form bubbles and overflowed, which caused spillage. When homogenization speed increased from 1000 to $7000 \mathrm{rpm}$ in $10 \mathrm{~min}$ (Figure S4, supplementary data), the overall average diameter of the nanoparticles was reduced from $325 \pm 1$ to $277 \pm 4 \mathrm{~nm}$. Figure S5 to S9 (supplementary data) shows the effect of homogenizer at same speed for different time intervals on behaviour of chitosan nanoparticles. Increasing time of homogenization further from $10 \mathrm{~min}$ to $25 \mathrm{~min}$ caused nanoparticles size to be reduced, however, this again caused bubble formation. Chitosan showed consistent and reproducible formulation in the terms of size distribution of nanoparticles, but high shear force greatly impacted polydispersity and zeta potential values. However, considering the effect of homogenization speed and time, on the results based on distribution of particle size, method consistency and production yield, it was difficult to determine the optimum formulation. As a result of the uncertainty about the optimum formulation, all four formulations were selected for protein loading and in vitro process protein release study to allow further formulation development and optimization.

The size of the silica nanoparticles also decreased from $246 \pm 2$ to $185 \pm 2 \mathrm{~nm}$ in silica sample SNPs (Figure S5 to S9, supplementary data) with different homogenizer speeds at fixed time, however, increasing homogenization time did not greatly affect the particle size as shown in (Figure S7, supplementary data) (184 \pm 76 to $173 \pm 11 \mathrm{~nm})$. On the other hand, PDI was greatly affected after homogenization and simultaneously zeta potential value also increased which helped to increase the monodispersed nature of the particles in solution. The PDI at $1000 \mathrm{rpm}$ was $0.34 \pm 0.01$, but increasing homogenizer speed to 10,000 caused the PDI to be reduced to $0.21 \pm 0.02$. This was evidenced by changing from bimodal at the low speed to monomodal at the higher homogenization speed. Zeta potential value increased from $-27 \pm 2$ to $-35 \pm 1$ in silica nanoparticles (Figure S6, supplementary data), which indicated that particles stability in solution was increased. It was difficult to carry out homogenization beyond $25 \mathrm{~min}$ at 10,000 speeds due to bubble formation, therefore homogenizer speed of 10,000 with $25 \mathrm{~min}$ homogenization time was deemed optimised for further formulation development.

Overall, the effect of homogenization speed (at fixed time of stirring) and different durations (at fixed speed) appeared to be more pronounced on size and PDI of PLGA nanoparticles compared to the chitosan and silica nanoparticles as shown in figures S5, S6 and S7, whilst the differences in effect on zeta potential between the three types of formulations was less pronounced (figures S8 and S9. 


\subsection{Effect of ultra-sonication}

Figure 3a, illustrates the effect of sonication on particle size in the PLGA, chitosan and silica nanoparticles. The results in Figure 3 showed that probe sonication clearly disintegrated the initial particles and formed smaller and more monodisperse sizes.

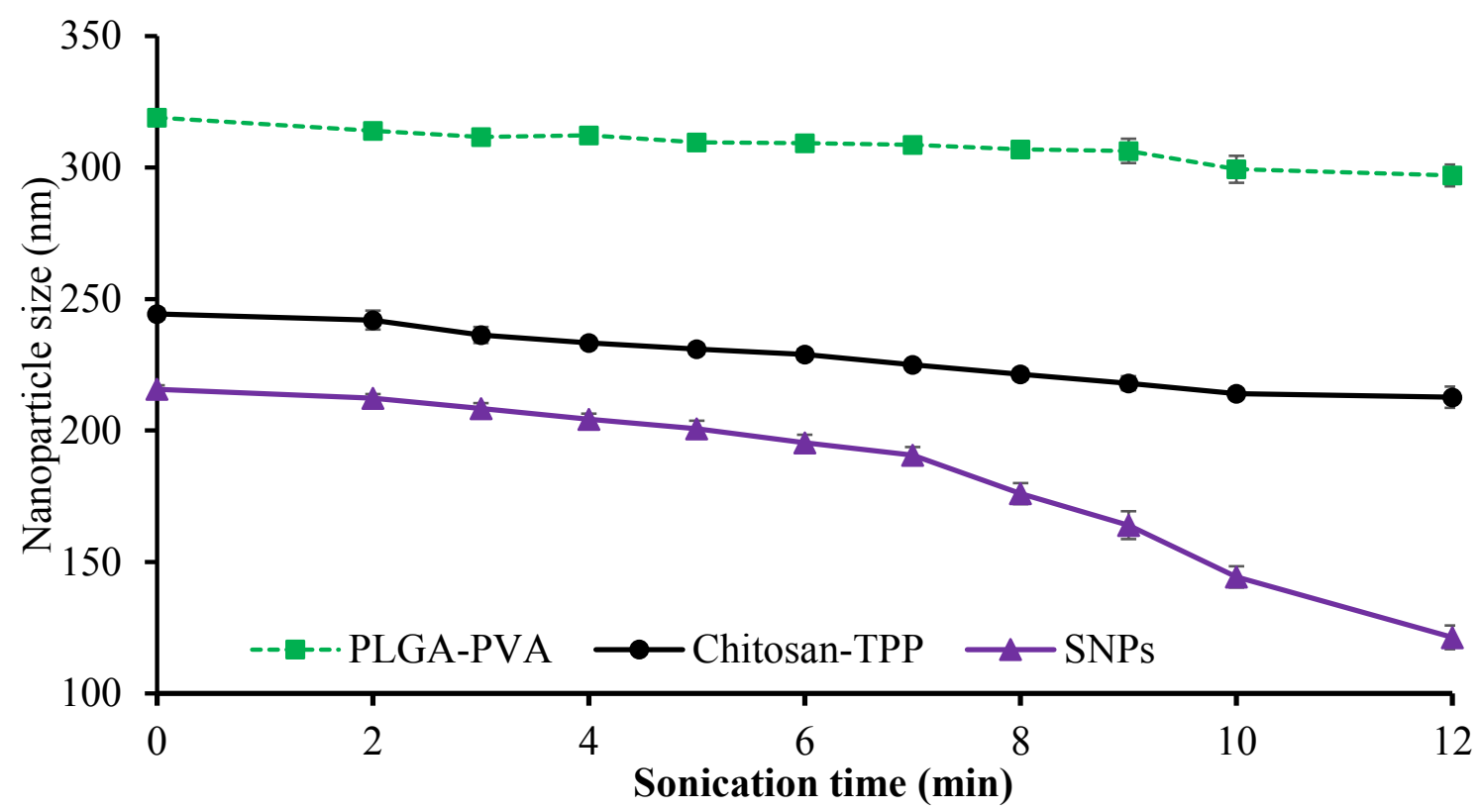

Figure 3 Investigation of (a) effect of ultra-sonication on size of selected optimised blank poly(lacticco-glycolic acid)-polyvinyl alcohol (PLGA-PVA), chitosan-sodium tripolyphosphate (TPP) and silica (SNPs) nanoparticles and (b) effect of ultra-sonication on zeta potential of selected optimised blank poly(lactic-co-glycolic acid)-polyvinyl alcohol (PLGA-PVA), chitosan-sodium tripolyphosphate (TPP) and silica (SNPs) nanoparticles $(n=3)$.

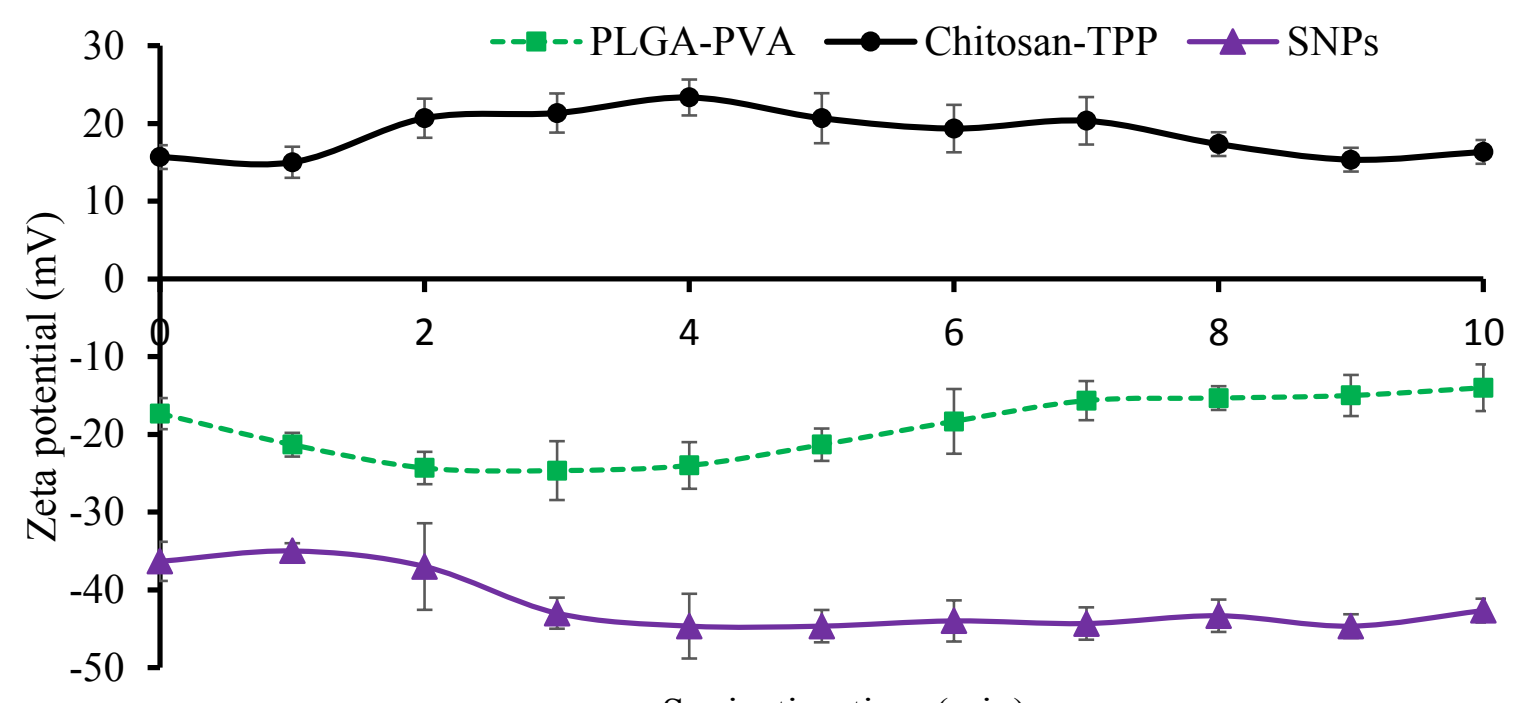

Sonication time (min)

Figure $3 \mathrm{~b}$ : Investigation of effect of ultra-sonication on zeta potential of selected optimised blank 
poly(lactic-co-glycolic acid)-polyvinyl alcohol (PLGA-PVA), chitosan-sodium tripolyphosphate (TPP) and silica (SNPs) nanoparticles $(n=3)$.

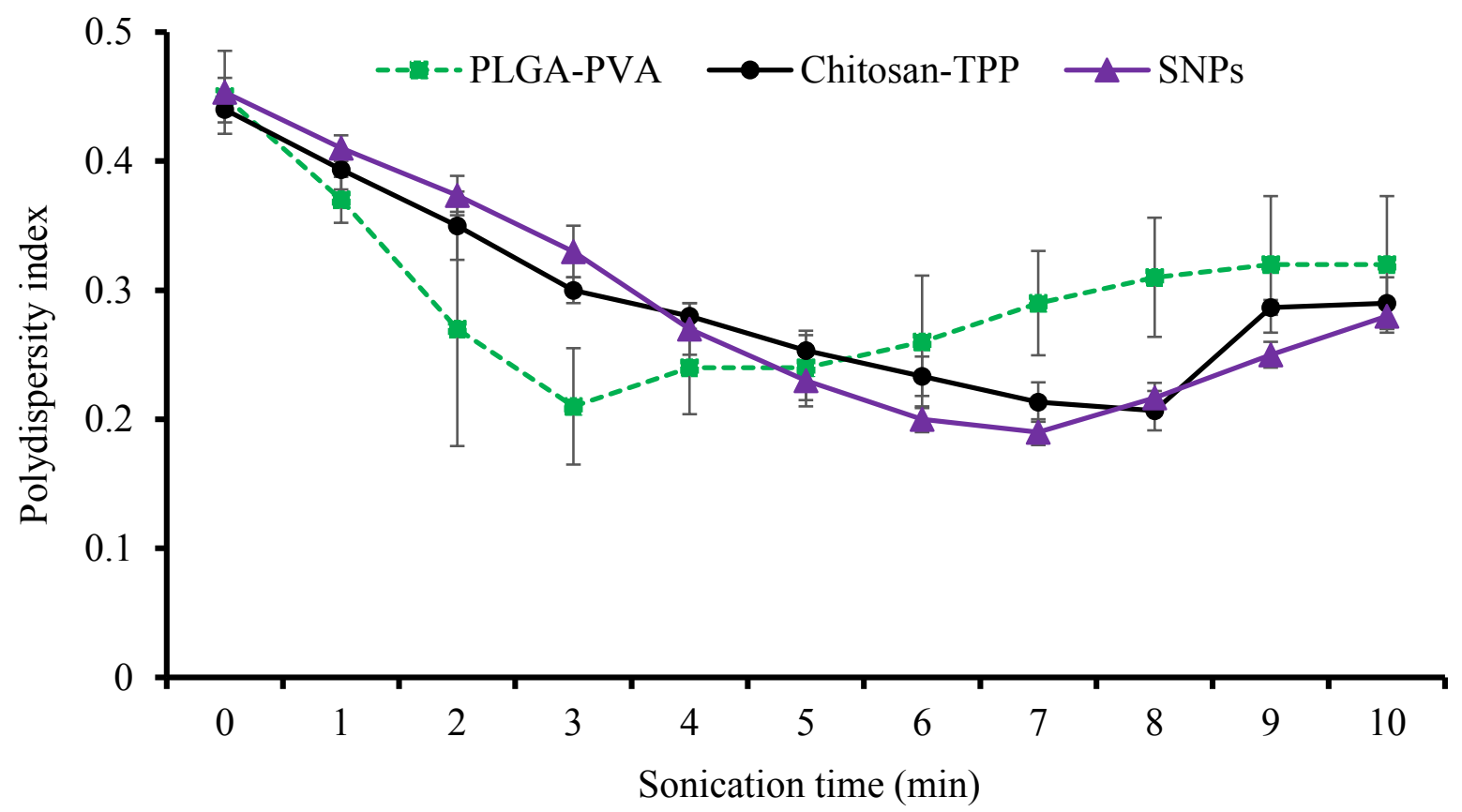

Figure 3c: Investigation of effect of sonication on PDI of selected optimised blank poly(lactic-coglycolic acid)-polyvinyl alcohol (PLGA-PVA), chitosan-sodium tripolyphosphate (TPP) and silica (SNPs) nanoparticles $(n=3)$.

PDI graph (Figure 3c) for PLGA-PVA formulation showed that within 2 min of probe sonication, the particles became monodispersed and PDI decreased subsequently. This finding is consistent with other research strategy previously reported [8]. However, further probe sonication beyond 2 min reduced particles size more and after 5 min, the PDI values increased significantly $(p>0.05)$ again while zeta potential (Figure $3 b)$ values decreased $(p>0.05)$.

The other two PLGA formulations prepared using different surfactants (PLGA-TW and PLGA-PL), showed a significant effect $(p<0.05)$ of sonication on their particles size, PDI and zeta potential values. Further, sonication in both formulations caused zeta potential value to be reduced and might be due to its effect on the nanoparticles structure and subsequent agglomeration. The results (Figures S10, S11, S12, supplementary data) showed relatively small particle agglomeration during a prolonged probe sonication and slight increase in zeta potential until 4 min after which no significant $(p>0.05)$ impact was observed in zeta potential and PDI values. It was therefore concluded that the nanoparticles size reduced, were monodisperse and stable within 4 min of sonication. These results are expected since the high concentration of surfactant (PVA, PL or TW) leads to high collision frequency of NPs, and therefore a high probability of agglomeration. Similar observation has been reported for TiO2's NPs (Tantra et al. 2015).

The average diameter of chitosan nanoparticles was $265 \pm 1 \mathrm{~nm}$, PDI at $0.31 \pm 0.05$ and zeta potential was $19 \pm 1 \mathrm{mV}$ at $40 \%$ amplitude. In Figure 3a, average diameter of chitosan nanoparticles 
decreased after sonication, but the particle size reduction rate after $8 \mathrm{~min}$ of sonication was not significant $(p>0.05)$. The DLS analysis indicates the reduction of the average particle size from 245 to $215 \mathrm{~nm}$ when ultra-sonication time increased from 2 to $10 \mathrm{~min}$. Zeta potential value in Figure $3 \mathrm{~b}$ also increased while PDI value decreased between $1 \mathrm{~min}$ and $4 \mathrm{~min}$ of sonication time. The reduction in size of the particles after ultra-sonication was probably related to the depolymerisation of the chitosan molecules. This is because the change in the average particle diameter of the nanoparticles was almost similar even after changing the ultra-sonication time.

There was a high tendency for agglomeration when the silica nanoparticles were prepared using the sol-gel synthesis method. According to recent studies [27], other methods used to enhance both nano fluid dispersion and stability are inadequate because these procedures only take very dilute volume fractions and also depend on the opacity of the particles dispersed. With increased ultra-sonication time it was shown that particles size was decreased in sample PLGA 1 to PLGA5 which reduced the extent of agglomeration. Unlike PLGA and chitosan, the average particle size (Figure 1A) of the silica nanoparticles decreased after ultra-sonication for $6 \mathrm{~min}$, PDI (Figure 1B) also decreased within the same time period.

Unlike homogenization, where PLGA nanoparticles were most affected, probe sonication affected the silica nanoparticles compared to the optimised PLGA and chitosan nanoparticles. The silica nanoparticles showed significant reduction in particle size below 200nm after 6 minutes of sonication, whilst the PLGA and chitosan nanoparticles remained far higher than $200 \mathrm{~nm}$ even after 12 minutes of probe sonication. This suggests that different energy input affects different chemical nano-structures in a different way.

\subsection{BSA loading efficiency of nanoparticles}

Figure 4 shows the protein loading and encapsulation efficiencies of selected optimised nanoparticles of the different formulations (PLGA, chitosan and silica) investigated.

In protein based vaccine delivery research for PLGA nanoparticles, the preferred size is 200 to $500 \mathrm{~nm}$ and at this dimension, nanoparticles can easily trigger dendritic cells (DCs), antigen specific $\mathrm{T}$ helper cells and cytotoxic $\mathrm{T}$ lymphocytic cells in order to activate cellular immune response and they can be endocytosed by antigen presenting cells (APCs) [28]. Figure 4 showed that blank PLGA-1\% PVA nanoparticle which had an original size of $244 \mathrm{~nm}$, increased in size to $292 \mathrm{~nm}$ after protein loading. 

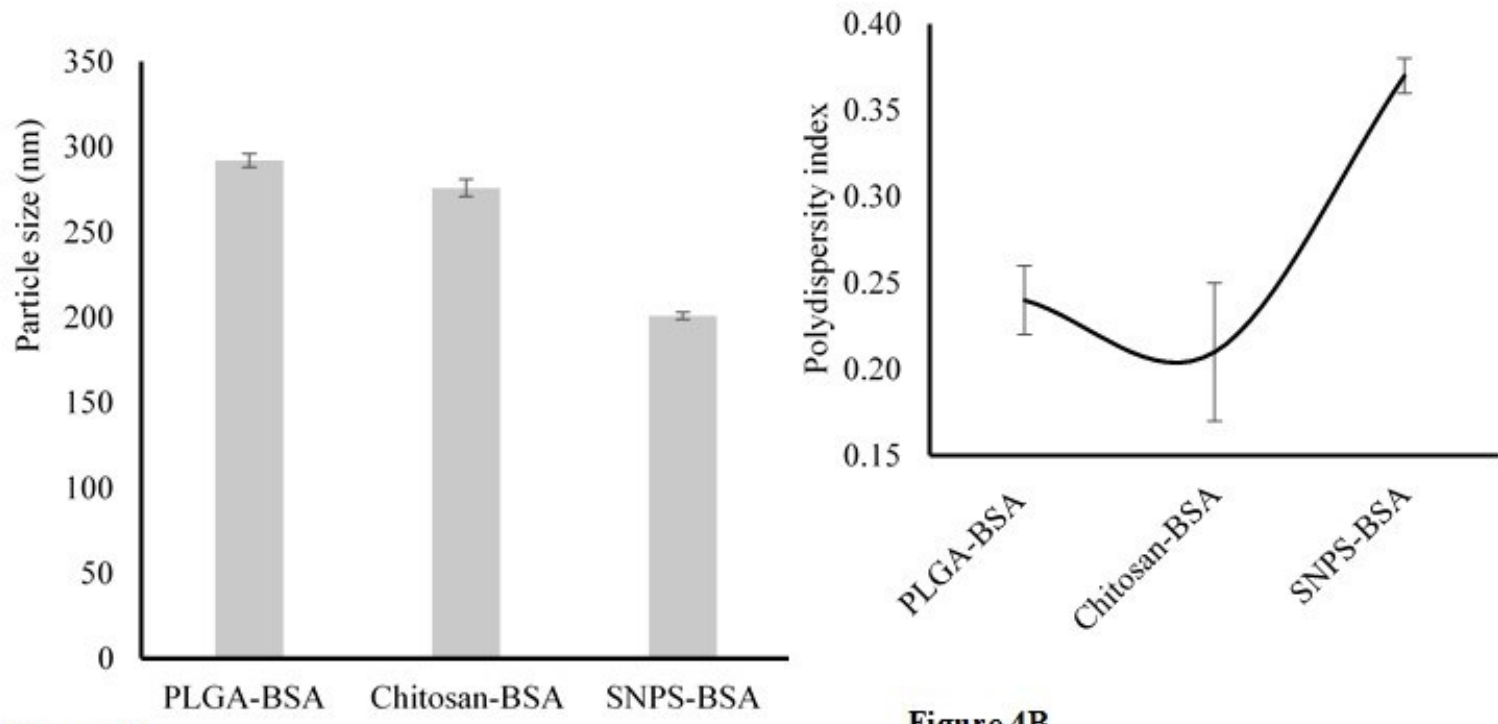

Figure 4A

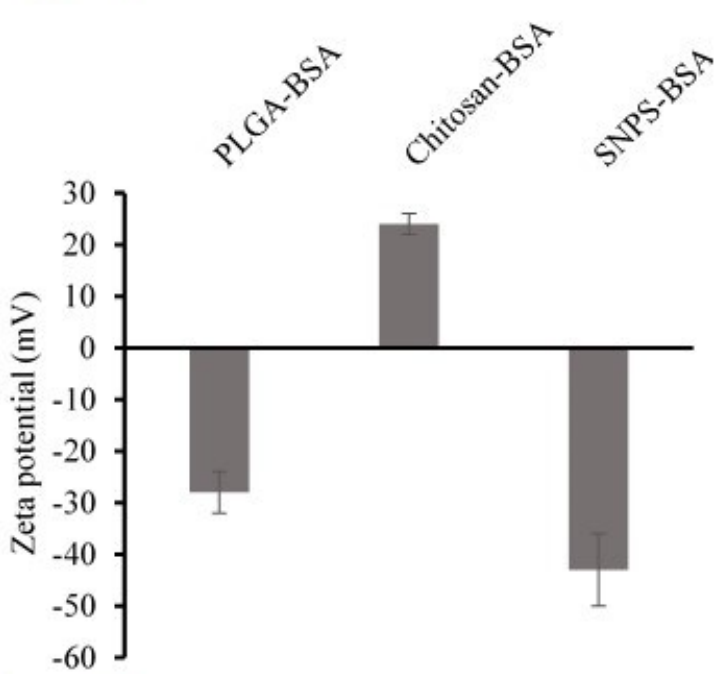

Figure 4C

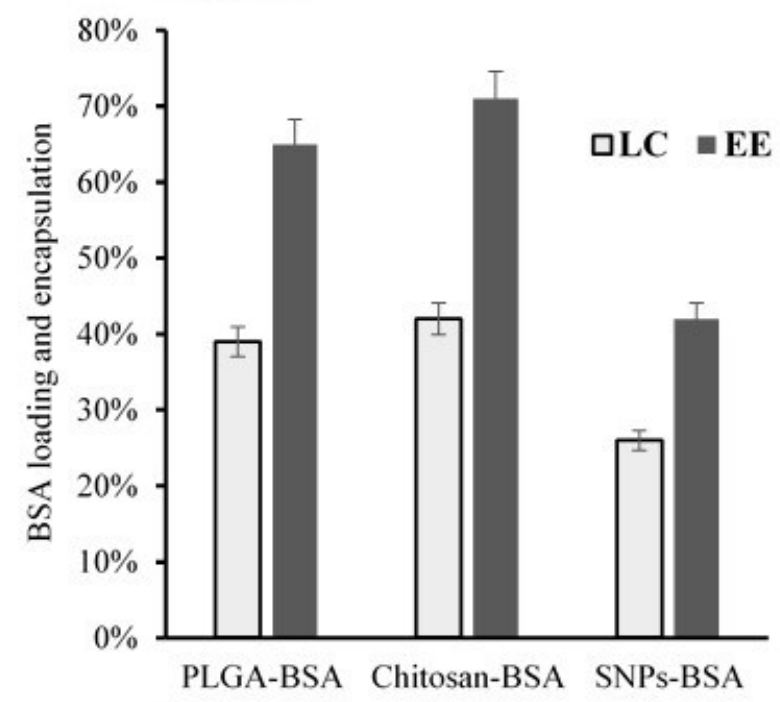

Figure 4D

Figure 4: Bovine serum albumin (BSA) (model protein) loading capacity (LC) and encapsulation efficiency (EE) in the formulated poly(lactic-co-glycolic acid) (PLGA), chitosan and silica (SNPs) nanoparticles $(n=3)$.

The same blank formulation showed zeta potential of $-17 \pm 6 \mathrm{mV}$ and PDI of $0.36 \pm 0.05$ but after BSA loading showed a zeta potential of $-28 \pm 3 \mathrm{mV}$ and PDI of $0.24 \pm 0.04$. PLGA-1.5\% PVA blank nanoparticle had a size of $278 \mathrm{~nm}$ but after protein encapsulation this was increased to $319 \mathrm{~nm}$ (Figure S14 supplementary data). In addition, protein loaded PLGA-PL, PLGA-TW formulations also showed differences from blank nanoparticle in terms of size. The value of zeta potential after protein loading suggests that most of the PVA based formulations were stable in the colloidal dispersion due to zeta potential values near -30 mV (Figure S16). However, the zeta potential values of PLGA-1\%TW and PLGA-1\%PL formulation suggest that these formulations should not be stored in liquid suspension 
form and but stored in a lyophilized state. The result of protein loading capacity and encapsulating efficiency in optimised PLGA-\%PVA formulation showed 63\% protein loading due to similar surface charge for both polymer and surfactant and high particle dispersion. However, the protein loading of all formulations were still lower than expected and indicates poor protein encapsulation during the nanoparticle preparation procedure. These findings also suggest that a high polymer concentration may be required as well as the need to decrease particle size which will increase surface area to help improve protein loading.

For chitosan, encapsulation of protein is largely the consequence of how the cationic chitosan chain interacts with protein molecules which have both positive and negatively charged functional groups and also hydrophobic regions in an aqueous environment. Some studies [29] suggest that, the encapsulation efficiency of protein is high irrespective of the chitosan molecular weight and indicated that the entanglement of the protein within the chitosan chain is not effected by their molecular weight.

The fixed BSA concentration at $0.5 \mathrm{mg} / \mathrm{ml}$ with formulations prepared with different chitosan concentrations $(0.1 \%$ to $0.4 \%$ ) showed that loading efficiency (LE) was increased from $68 \%$ to $72 \%$, similarly loading capacity (LC) also increased from 27 to $48 \%$ (Figure S13, supplementary data). After protein loading, the particles size also increased from $221 \pm 16$ to $246 \pm 27$ for $0.1 \%$ to $0.4 \%$ (Figure S14).

Profiles of silica nanoparticles encapsulating BSA, LC and LE are summarized in Figure S13 (supplementary data). The encapsulation of silica-6 sample was $25 \%$ due to low yield in the amount of nanoparticles formed as confirmed by DLS and SEM images while in sample silica-8, a maximum of $42 \%$ BSA encapsulation was obtained. In addition, the tendency of particle aggregation and agglomeration of nanoparticles was higher according to PDI value, though the weight ratio for encapsulation between proteins and MSNs was 1:1. Another important factor that affects encapsulation of proteins in silica nanoparticles is surface chemistry and pore size. Pore size blocking by surfactant would lower amount of protein that could be encapsulated and therefore needs to be optimal. Zeta potential value was also analysed before and after encapsulation. All the formulated silica nanoparticles had a negative surface charge and zeta potential value remained negative after addition of BSA (Figure S16). In sample silica-9, the particles size changed from $176 \pm 7$ to $201 \pm 5 \mathrm{~nm}$ after protein loading, PDI value also increased from $0.24 \pm 0.11$ to 0.37 (Figure S15) and zeta potential value changed from $-33 \pm 6$ to $-43 \pm 3 \mathrm{mV}$.

Comparing between the three types (synthetic organic, natural organic and inorganic) of nanoparticles, the PLGA and silica formulations had negative zeta potential compared to the chitosan which remained positive. Further, the silica particles appeared to have higher zeta potential values than the PLGA and chitosan particles suggesting the inorganic particles were more physically stable than the organic equivalents. This is interesting, given the fact that the silica nanoparticles remained small in size (around 200nm) compared to PLGA and chitosan, which increased in size above 300nm after protein loading. 


\subsection{Morphology of the nanoparticles by electron microscopy}

The morphology of formulated nanoparticles was determined through the visualization of their microscopic appearance using SEM and TEM.

Figure 5 showed that the PLGA nanoparticles were of spherical shape Figure 5 (A) represents PLGA-PVA $1.0 \%$ formulation, showing spherically shaped nanoparticles with size range between 200 $\mathrm{nm}$ to $1 \mu \mathrm{m}$. Figure 5(b) represents PLGA-PVA 1.0\% after sonication and showed smooth spherical shape but larger particles were also present. Figure 5(c), representing PLGA-PVA1\% formulation after BSA loading, was spherical in shape and porous with uniform dispersion of particles.

Figure 5 (d) shows chitosan $0.1 \%$ nanoparticles which showed a high number of particles formed but with varied sizes. Figure 5(e), represents chitosan $0.1 \%$ formulation after sonication and showed spherical shape, with size range between $200 \mathrm{~nm}$ to $1 \mu \mathrm{m}$ and ellipsoidal shapes of some of the particles. This probably results, from high speed homogenization and sonication, forming turbulences which induce a heterogeneous energy distribution in the emulsion system, which results in some particles starting to lose the spherical appearance and becoming irregular in shape. Figure 5(f), representing BSA loaded chitosan $0.1 \%$ shows that all particles were regularly shaped and size ranged between $200 \mathrm{~nm}$ to $1 \mu \mathrm{m}$ and some large particles also appeared. It might be due to the fact that high speed stirring results in vigorous particle collisions between them and therefore formation of regular shaped particles. Figure 5 (g), representing optimised silica 9, showed smooth surface and size range between $200 \mathrm{~nm}$ to $1.5 \mu \mathrm{m}$ and Figure 4(h), shows silica 9 formulation after sonication, while Figure 5(i) shows corresponding BSA loaded silica 9 nanoparticles. SEM images for the other formulations prepared during formulation development and optimization are shown in Figure S17 (supplementary data). 

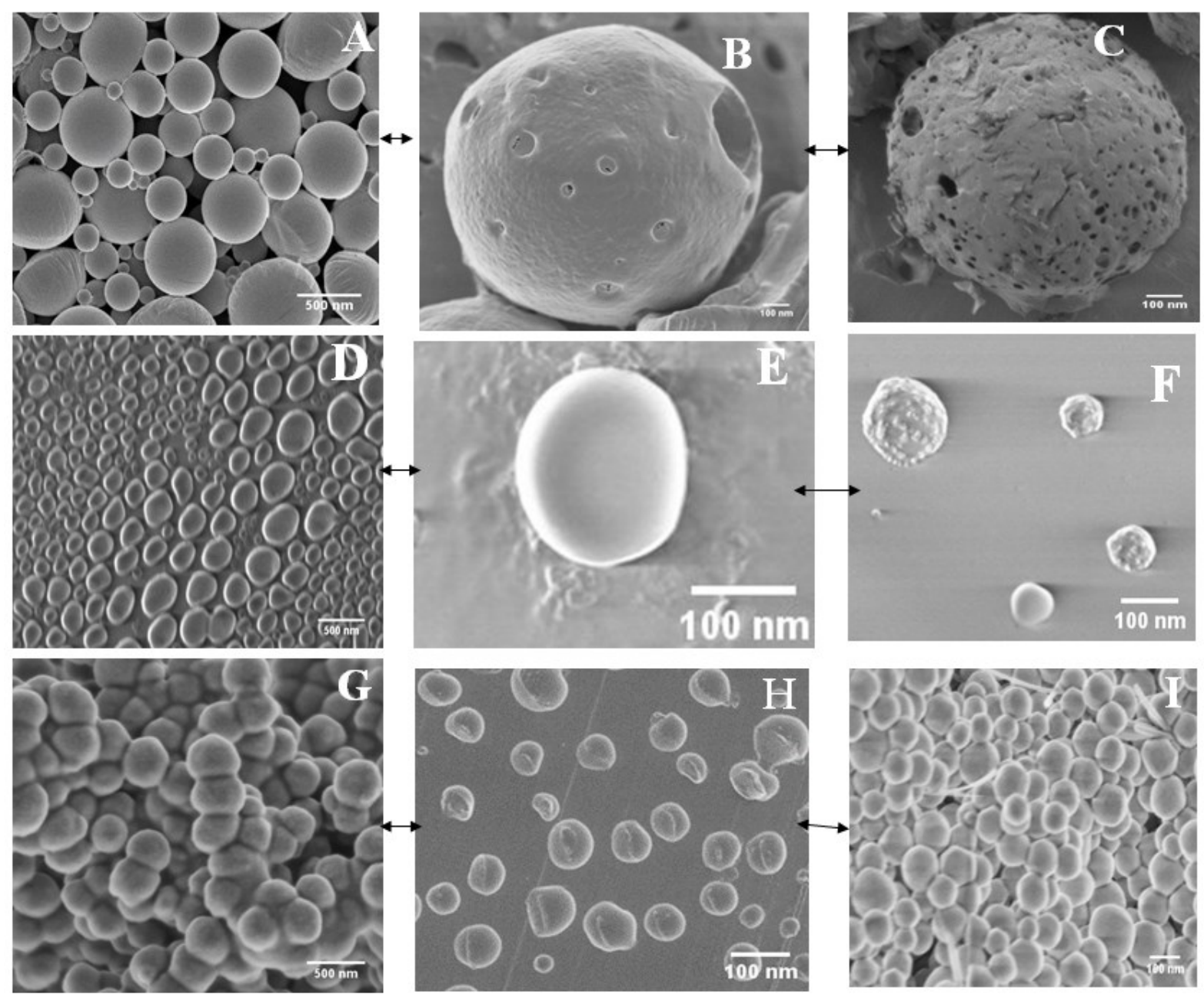

Figure 5: Scanning electron microscopy analysis of poly(lactic-co-glycolic acid) (PLGA), chitosan and silica nanoparticles. Image A; blank PLGA nanoparticles, Image B: sonicated blank PLGA nanoparticles, Image C; BSA loaded and sonicated PLGA nanoparticles, Image D; blank chitosan nanoparticles, Image E; blank sonicated chitosan nanoparticles, Image F; BSA loaded and sonicated chitosan nanoparticles. Image $\mathrm{G}$; blank silica nanoparticles, Image $\mathrm{H}$; sonicated blank silica nanoparticles, Image I; BSA loaded silica nanoparticles.

TEM and STEM allow the observation of liquid nanoparticle suspensions and might give an accurate idea about nanoparticle shape which allowed the visualization of BSA encapsulation within the PLGA nanoparticle. Figure S18 (supplementary data) showed the presence of a dense core in the nanoparticle embedded resin block with bright shell which confirms BSA loading within the PLGA polymeric matrix. The variations in brightness and contrast reflects the internal characteristic of the cross-sections of nanoparticles. In figure S18(a), the polymeric background encapsulating the BSA protein showed uniform and moderate brightness and contrast. The BSA-encapsulated areas are characterized by the dark colour showing a high contrast with the background. Figure S18(b), showed that the particles were closely aggregated with each other after protein encapsulation and Figure S18(c) 
confirmed that particle sizes were varied but not aggregated with each other. The uniform blank polymeric background showed in Figure S18(d) also demonstrated highlighted dark coloured area present. These BSA containing nanoparticles showed that the protein occupying areas were intersected and were visually porous in structure as confirmed by SEM images. Scanning transmission electron microscopy (STEM) image [Figure S18(f)] showed particles are uniformly dispersed but sizes were visually variable as confirmed by SEM image.

The size distribution of protein loaded chitosan-TPP nanoparticles obtained from STEM analysis is shown in Figure S19 (supplementary data). From the STEM images it can be observed that, the particle size of nanoparticles was slightly smaller than the equivalent size data obtained from DLS analysis. This is because DLS measures the hydrodynamic diameter of particles whereas SEM measures whole morphology, size and shape of nanoparticles. The STEM data indicates that the protein loaded chitosan nanoparticles' $(0.1 \%$ to $0.4 \%)$ size ranged between $100-200 \mathrm{~nm}$. A homogenous distribution of nanoparticles can be observed in Figure S19 (supplementary data) from the STEM analysis, whilst the SEM images also showed smooth and spherical shape of nanoparticles. The STEM images in Figures S19 showed BSA loaded chitosan-TPP nanoparticles with large particles present along with a large number of smaller particles. At high magnification, it was observed that the micron sized particles were actually clusters of agglomerated nanoparticles. Therefore, the micron-size images and nano-sized image was scanned by STEM with a magnification of 5k and 20k. From Figures S20, S21, S22 and S23 (supplementary data) for silica-CTAB9 and silica-CTAB10, it can be seen that there was no change in the general background of the STEM image (blank and protein loaded) before and after the radiation.

\subsection{Attenuated total reflectance-Fourier transform infrared spectroscopy (ATR-FTIR) ATR-}

FTIR analysis was used to determine specific functional groups or chemical bonds in the samples by the vibration modes of these bonds when exposed to infrared radiation. The ATR- FTIR spectra obtained for pure starting materials is shown in Figure 6a whilst spectra for selected optimised blank and BSA loaded PLGA, chitosan and silica nanoparticles are shown in Figure 6b. Detailed ATR-spectra for all the formulations prepared as part of formulation development are shown in the supplementary data (Figures S24, S25, S26, S27 and S28) The strong band observed in the region between $1650 \mathrm{~cm}^{-1}$ and $1750 \mathrm{~cm}^{-1}$, can be attributed to the carbonyl group present in the formulated blank PLGA-PVA formulation. The broad band at $3300 \mathrm{~cm}^{-1}$ to $3600 \mathrm{~cm}^{-1}$ is attributed to the presence of $(\mathrm{OH})$ group of glycolide and lactide copolymer and from moisture content. PLGA-1\%PVA BSA loaded nanoparticles showed structural modification compared to blank nanoparticle with a major shift observed at $3450 \mathrm{~cm}^{-}$ ${ }^{1}$. The spectra of BSA loaded and blank PLGA nanoparticle showed all the characteristic peaks at 750$1250 \mathrm{~cm}-1\left(\mathrm{C}-\mathrm{C}, \mathrm{C}=\mathrm{O},-\mathrm{OCH}_{3}\right)$ and with negligible shift at $3305 \mathrm{~cm}^{-1}, 3294 \mathrm{~cm}^{-1}, 2886 \mathrm{~cm}^{-1}, 2927 \mathrm{~cm}^{-}$

1, $1749 \mathrm{~cm}^{-1}, 1343 \mathrm{~cm}^{-1}$ and $852 \mathrm{~cm}^{-1}$, which indicated there was no significant chemical structural change for blank PLGA during BSA loading. The FTIR spectra of pure BSA compared with the protein 
loaded nanoparticle showed that the $1600-1800 \mathrm{~cm}^{-1}$ band, indicating $\mathrm{C}=\mathrm{C}$ stretching, was significantly lowered and suggests that BSA was largely incorporated into the nanoparticle matrix.

ATR-FTIR spectroscopy was also employed to identify any modification that occurred during the chemical transformation between chitosan, TPP and BSA. In $0.4 \%$ chitosan formulation (Figure S26, supplementary data) a peak can be observed at $1412 \mathrm{~cm}^{-1}$, which indicates $-\mathrm{NH}_{2}$ bending vibration which might be attributed to the phosphoric group of TPP linked with $\mathrm{NH}_{3}{ }^{+}$group of chitosan suggesting that inter and intra molecular interaction are enhanced in chitosan-TPP nanoparticles. BSA loaded $0.1 \%$ chitosan formulation showed a sharp peak at $1076 \mathrm{~cm}^{-1}$ corresponding to the stretching vibration of the $\mathrm{P}=\mathrm{O}$ groups in chitosan-TPP nanoparticles.

For silica nanoparticles the vibrating band between $400-1200 \mathrm{~cm}^{-1}$ is attributed to $\mathrm{Si}-\mathrm{O}-\mathrm{Si}$ vibrations, and having a shoulder at $1190 \mathrm{~cm}^{-1}$. The spectra of BSA loaded silica nanoparticles demonstrates the presence of extra bands at $2985 \mathrm{~cm}^{-1}$ and $2960 \mathrm{~cm}^{-1}$ attributed $\mathrm{C}-\mathrm{H}$ bonds asymmetric and symmetric stretching vibrations, an extra band at $2947 \mathrm{~cm}^{-1}$ attributed to the methyl group connected to a nitrogen atom of BSA loaded nanoparticles. It was observed that between 1500 and $1300 \mathrm{~cm}^{-1}$, two vibration bands can be distinguished, which can be attributed to BSA loading. The $1451 \mathrm{~cm}^{-1}$ band is attributed to the $\mathrm{C}-\mathrm{H}$ deformation vibration and another band at $1398 \mathrm{~cm}^{-1}$ due to stretching vibrations indicating $\mathrm{CH}_{2}$ bending. 


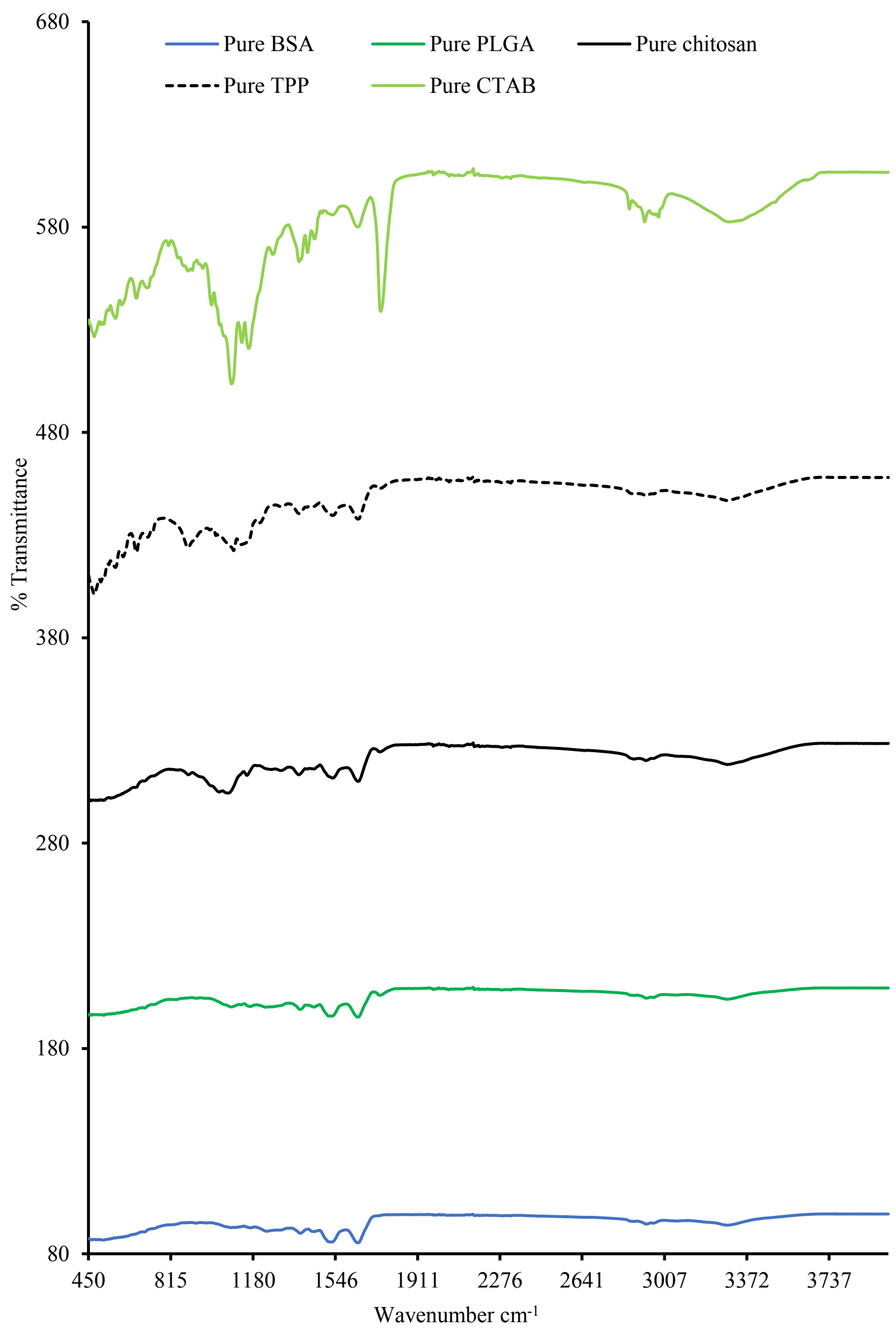

Figure 6a: FTIR spectra of pure starting materials - bovine serum albumin (BSA), poly(lactic-coglycolic acid) (PLGA), cetyltrimethylammonium bromide (CTAB), and tripolyphosphate (TPP). 


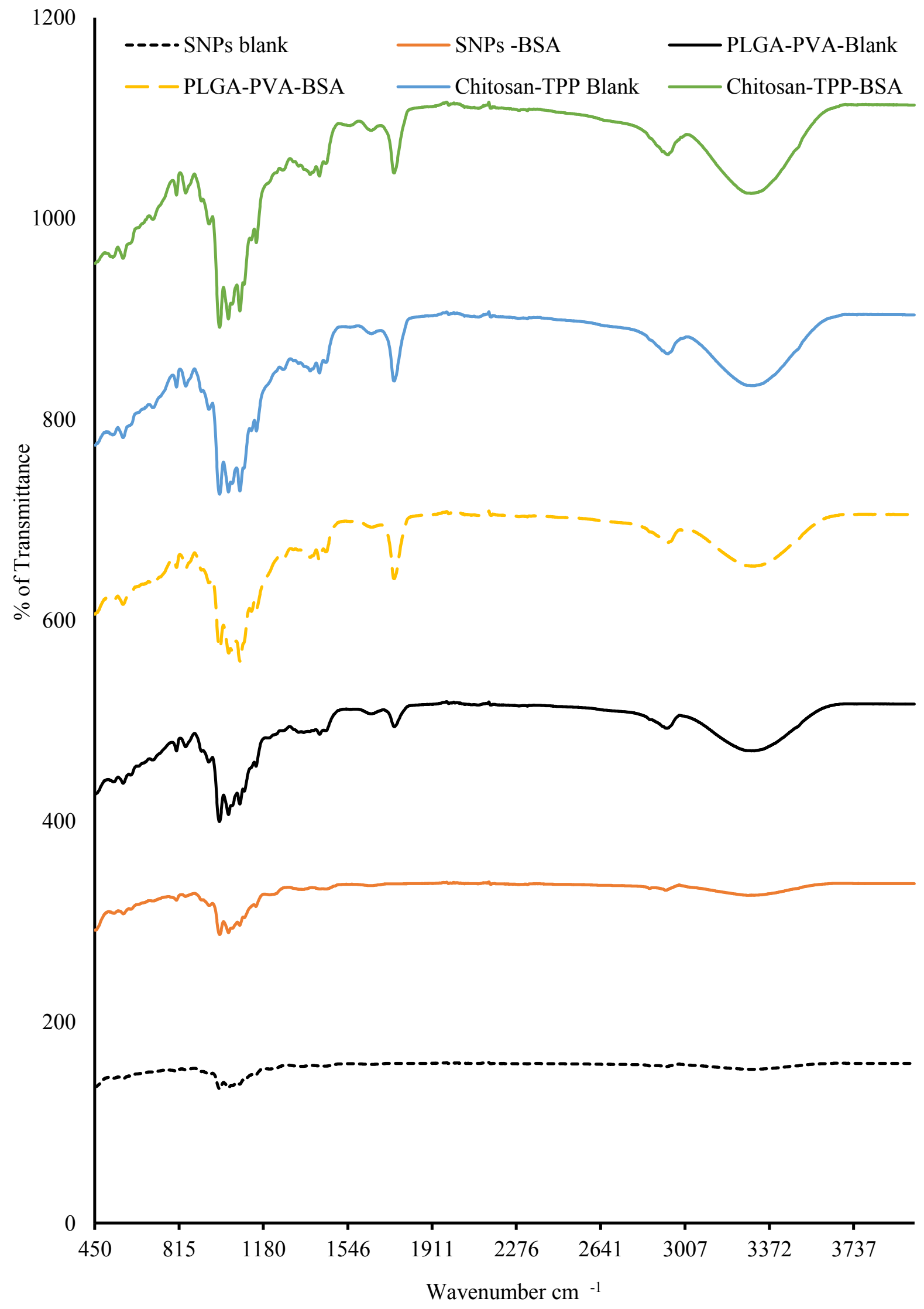

Figure 6b: ATR-FTIR analysis of blank and bovine serum albumin (BSA) loaded poly(lactic-coglycolic acid)-polyvinyl alcohol (PLGA-PVA), chitosan-tripolyphosphate (chitosan-TPP) and silica (SNPs) nanoparticles. 


\subsection{X-ray diffraction (XRD)}

The X-ray diffraction patterns of blank and BSA loaded nanoparticles were investigated to confirm the physical form (amorphous or crystalline nature) of the nanoparticles and shown in Figures 7a and $7 \mathrm{~b}$. PLGA-1\%PVA showed crystalline nature after BSA was added but with reduced crystallinity and the sharp intense peak shown at $24^{\circ} 2 \theta$ might be due to the presence of trehalose in the matrix. Comparing BSA loaded PLGA-PVA (Figure S29, supplementary data) nanoparticles with the corresponding blank nanoparticles, there was a reduction in intensity and number of peaks after protein loading, which indicates reduced crystallinity. Blank PLGA-1.5\%PL (Figure S30, supplementary data) nanoparticle formulation exhibited reduced crystallinity and most intense peaks occurred at diffraction angle of $24^{\circ}$ 20. BSA loaded PLGA-1.5\%PL (Figure S30, supplementary data) nanoparticle showed no sharp peak and indicates that the trehalose dispersed in the polymer and was possibly converted from crystalline to amorphous form after freeze drying. PLGA-1.5\%TW (Figure S30, supplementary data) nanoparticle formulation showed peaks with similar intensity to the blank nanoparticle which might be due to the high amount of trehalose present in blank nanoparticles in this formulation as discussed above.

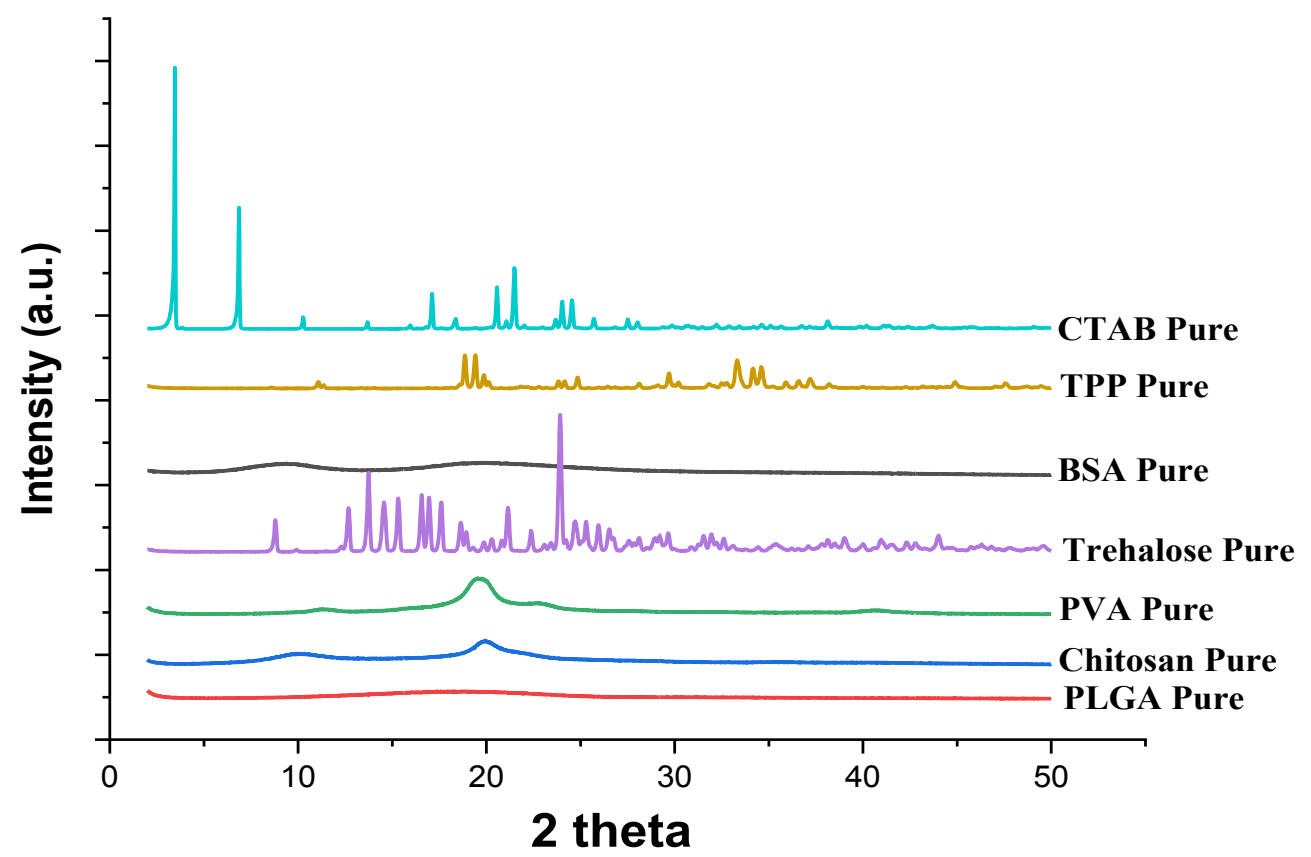

Figure 7a: XRD-diffractograms of pure starting materials - bovine serum albumin (BSA), cetyltrimethylammonium bromide (CTAB), chitosan, polyvinyl alcohol (PVA), poly(lactic-co-glycolic acid)(PLGA), trehalose and tripolyphosphate (TPP). 


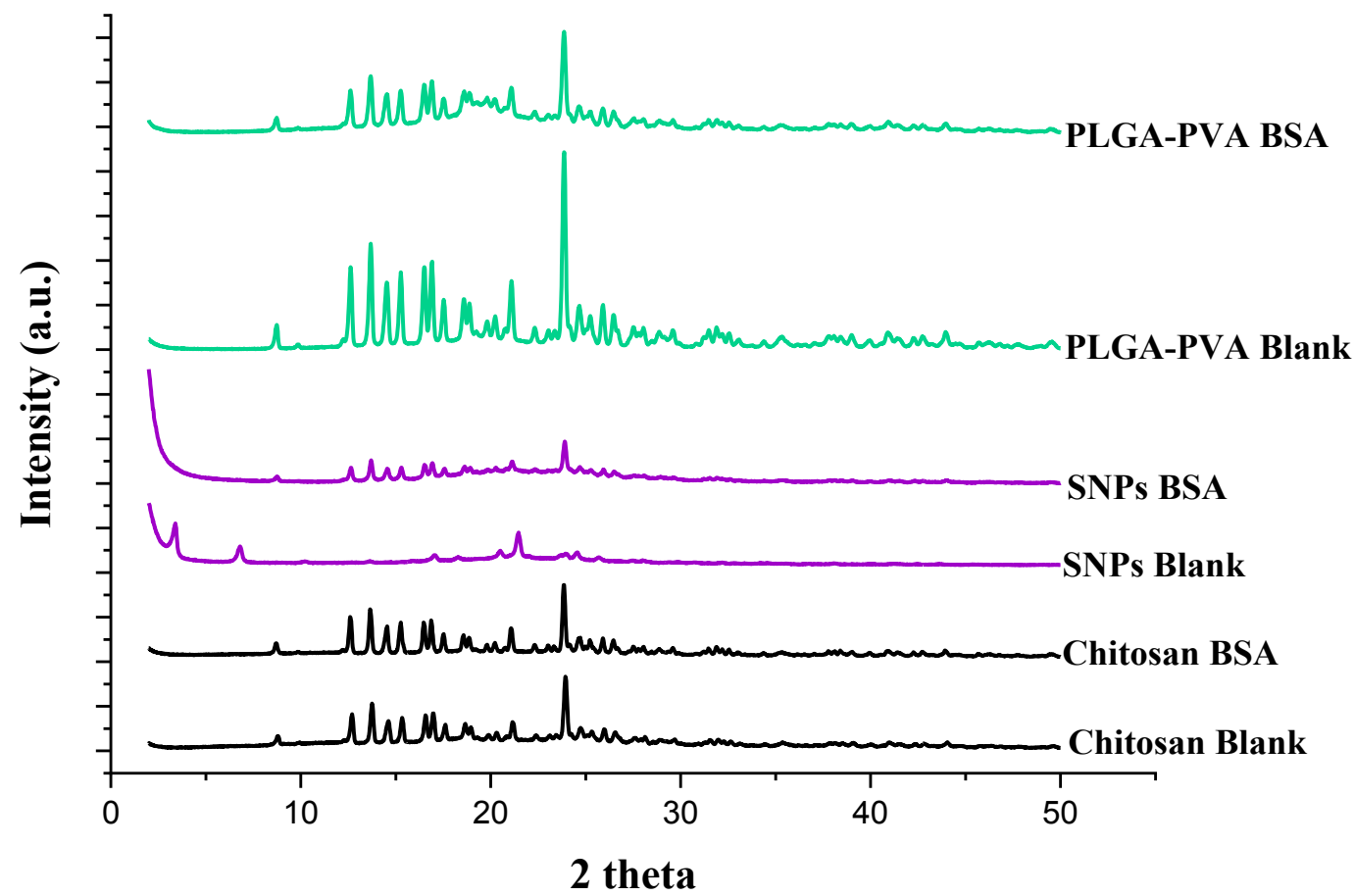

Figure 7b: XRD-diffractogram of blank and bovine serum albumin (BSA) loaded poly(lactic-coglycolic acid)-polyvinyl alcohol (PLGA-PVA), chitosan and silica (SNPs) nanoparticles.

The pure chitosan was in amorphous state (Figure 7a) but after nanoparticle formation and encapsulation of BSA protein, a crystalline phase was observed (Figure 7b). The XRD patterns of BSA loaded chitosan-TPP nanoparticles showed a narrow intensity peak at $24^{\circ}$, characterizing the crystalline state of all the BSA loaded chitosan-TPP nanoparticles (Figure 7b). This is due to the interaction between crystalline TPP and chitosan, but with reduced crystallinity. The positions of the narrow intensity peaks was not shifted in the BSA loaded nanoparticles (compared to blank) but peak intensity reduced after BSA loading suggesting a certain degree of interaction between BSA and chitosan-TPP nanoparticles [30]. Hydrogen bonding is formed based on the nanoparticle complexes between the nitrogen atoms of BSA protein and $\mathrm{H}$ atoms from $\mathrm{NH}_{2}, \mathrm{NH}_{3}{ }^{+}$and $\mathrm{P}=\mathrm{O}$ groups of chitosan-TPP nanoparticles. Several researchers [31][32] also reported a similar phenomenon, with reduction of crystalline peaks after protein encapsulation due to interaction between BSA and chitosan. The XRD patterns of the other formulated chitosan nanoparticles are shown in Figures S31 and S32.

Figure $7 \mathrm{~b}$, indicates that the conjugated and entrapped forms of the protein are unable to form their own crystal lattice inside the polymer and therefore are in amorphous state [33]. These results are interesting since in the amorphous state, the molecules are arranged randomly, which results in lower energy consumption and dissolution rate is faster, thereby the potential of increasing the protein bioavailability. 
The XRD pattern of silica nanoparticles showed three considerable peaks corresponding to lattice spacing of 20.631, 21.403 and 21.675 (Figure $7 b$ ) which compares very well with XRD diffractogram for $\mathrm{SiO}_{2}$. Figure (S33, S34, supplementary data) shows the XRD patterns of silica nanoparticle samples Silica6, Silica7, Silica8, Silica9, and Silica10. The results show that all BSA loaded and blank silica samples were relatively amorphous in nature and results are consistent with that reported by others (Liao et al., 2014).

\subsection{In vitro protein release study:}

In vitro protein release study was performed in phosphate buffer $(\mathrm{pH} 7.4)$ in order to determine the capability of optimised BSA loaded nanoparticles to release encapsulated protein and Figure 8 shows the dissolution profiles for the selected optimised formulation for each type of nanoparticle being compared. Initial burst release (Figure 8) occurred for PLGA-1\%PVA nanoparticles with more than $25 \%$ of protein released within $4 \mathrm{hrs}$. On the other hand, for PLGA-1.5\% PVA (Figure S35, supplementary data) nanoparticles, about $20 \%$ protein was released after $6 \mathrm{hrs}$. The release patterns of PLGA-1\% PVA (Figure 89) and PLGA-1.5\% PVA formulation were very similar with an initial burst release within the first $2 \mathrm{hrs}$ and a sustained release pattern until $48 \mathrm{hrs}$, which is characteristic of PLGAbased nanoparticles [35].

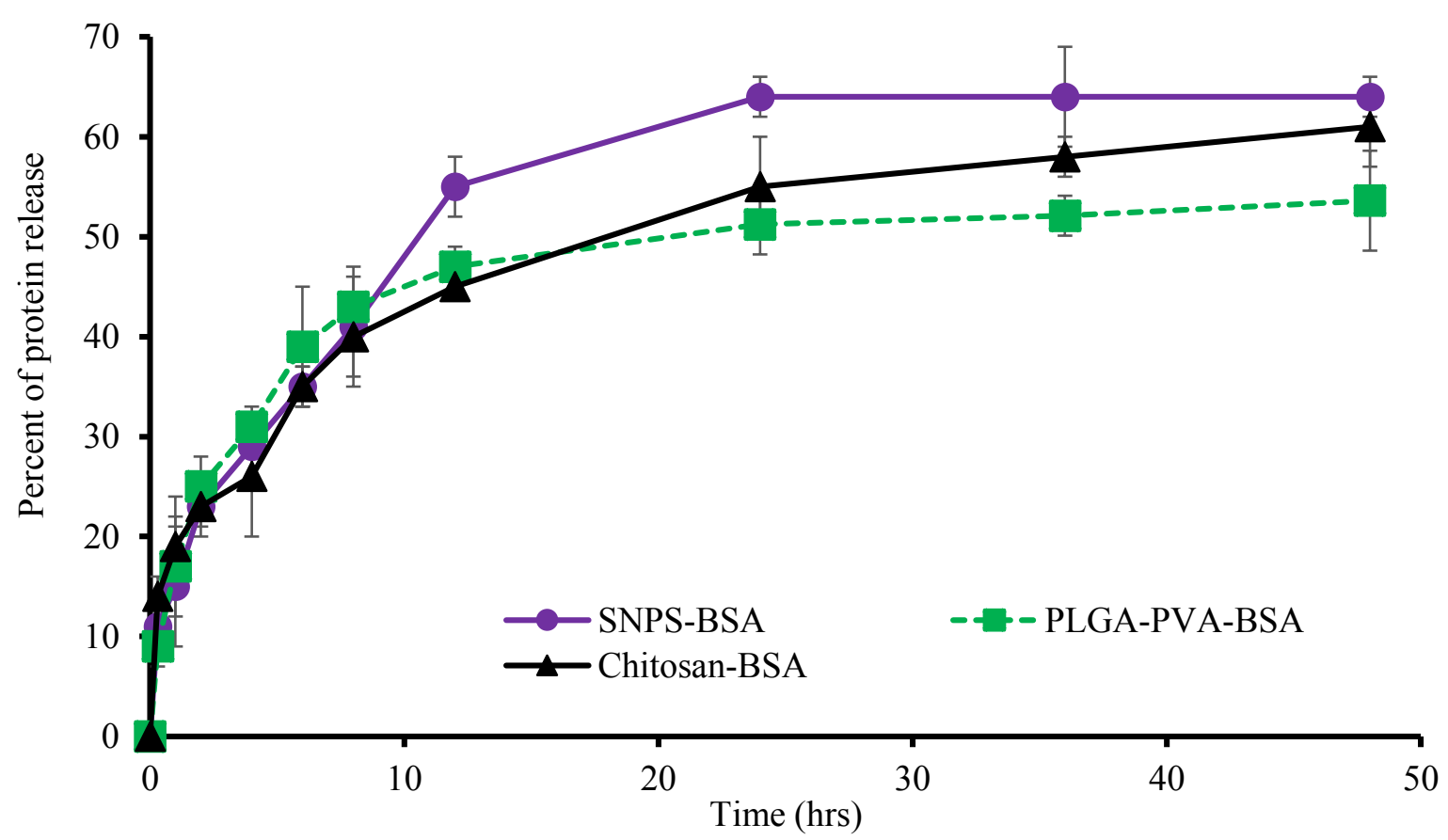

Figure 8: In-vitro BSA release study from poly(lactic-co-glycolic acid)-polyvinyl alcohol (PLGA-PVA) (PLGA-PVA), chitosan and silica (SNPs) nanoparticles $(n=3)$.

On the other hand, PLGA-1\%PL (Figure S35, supplementary data) nanoparticles showed more than $20 \%$ protein release after 2 hrs and about $40 \%$ within 8 hrs. PLGA-1\% TW (Figure S35, supplementary 
data) nanoparticles showed more than $40 \%$ protein release after $12 \mathrm{hrs}$. The initial burst release of protein from the nanoparticles may be explained by the BSA present on nanoparticle surface after which the encapsulated BSA was released over a longer time period and achieving sustained release pattern. In this study, it was shown that most of the nanoparticle formulations released 40 to $50 \%$ of protein within $24 \mathrm{hrs}$ in a sustained manner. For example, the cumulative amount of protein released from the PLGA-1\%PL (Figure S35, supplementary data) nanoparticles within 24 hrs was approximately 50\% whilst PLGA-1\%TW (Figure S35, supplementary data) was about $40 \%$.

PVA and PL could possibly delay the degradation of PLGA by neutralizing the acidity generated during polymer degradation and able to prevent unexpected interaction between polymer and protein, resulting in slow release of BSA from the polymer matrix [z1하] . It is possible that higher amounts of surfactant with low amount of polymer in PLGA-1\%PL nanoparticles and subsequent reduction in particle size of $192 \mathrm{~nm}$ and higher PDI of 0.31 (Figure S14, supplementary data) resulted in higher protein release due to increase in the surface area of the nanoparticle. Biodegradability and biocompatibility of PLGA nanoparticle is one of the most important properties for delivering biologically active molecules such as protein, peptide and small molecule drugs. Several studies have shown that, encapsulation of biologically active molecules into PLGA nanoparticles increased their bioavailability and reduced toxicity [37]. Sustained release of proteins from nanoparticle is imperative as that could provide long term therapeutic effect against diseases [38]. In some studies, it has been shown that high burst release especially within $24 \mathrm{hrs}$ is not preferred since it causes low T-cell response and antigenic protein release from nanoparticle in low burst fashion may achieve a better vaccine activity and immune response [39].

Nearly $68 \%$ loaded protein was completely released from chitosan nanoparticles in $48 \mathrm{hrs}$. Formulation $0.1 \%$ showed an initial burst release occurring within $2 \mathrm{hrs}$, with more than $19 \%$ of protein released within this time. Protein release for formulation $0.2 \%$ to $0.4 \%$ (Figure S36, supplementary data) increased sharply initially and slowed down up to $48 \mathrm{hrs}$. The highest protein released obtained from formulation $0.2 \%, 0.3 \%$ and $0.4 \%$ (Figure S36, supplementary data) after $48 \mathrm{hrs}$ was $62 \%, 64 \%$ and $52 \%$ respectively. As the concentration of chitosan increased the viscosity of the solution was increased which could result in strong particle walls, resulting in lower swelling ability, which therefore reduces the rate of protein release. In addition, there are electrostatic interaction between chitosan-TPP and BSA that could affect the percentage released. For smaller size nanoparticles, a greater percentage of BSA was released than from large particles because small particles have a greater surface to volume ratio, and the mechanism at this stage is diffusion of BSA protein inside the nanoparticles due to the concentration gradient.

Figure 8 also shows that the release of proteins from silica nanoparticles prepared from TEOS and CTAB. Large amount of BSA released was observed from sample silica 10 (Figure S37, supplementary data). The profile for silica and PL formulation showed that $17 \%$ BSA was released within 30 min and after $4 \mathrm{hrs}$ almost $43 \%$ of BSA was released. This might possibly be due to less 
formation of nanoparticles and it is also possible that lower protein might be encapsulated into the nanoparticles due to the excess amount of surfactant which could block nanoparticles pore and prevent protein adsorption by nanoparticles. After 48 hrs, 64\% BSA released was observed for silica 6 sample. Overall, though the nanoparticles were prepared from three different starting materials (synthetic organic, natural organic and inorganic), all three selected optimised formulated nanoparticles (PLGA, chitosan and silica) showed similar sustained release profiles (Figure 8). This is interesting, and suggests that the properties of the drug and the medium environment, play an important role in determining drug release characteristics from nanoparticles prepared from different materials, however, further investigation will be required in this regard.

\section{Conclusions}

This research aimed to formulate and compare the functional performance characteristics of nanoparticles prepared from three different starting materials (organic and inorganic) and investigate factors that affect the achievement of optimal size and high protein encapsulation for potential mucosal vaccination. The results demonstrated that the surfactant: polymer ratio, homogenizer speed and duration (time) of homogenization, greatly influenced nanoparticle size and PDI. The mean particle size of formulated optimised PLGA nanoparticles was smaller than that reported by other researchers and noted to be optimal for protein encapsulation. In terms of zeta potential and size, PLGA-1\% PVA and PLGA-1\%PL were optimal formulations for further protein encapsulation study. Both formulations exhibited sustained release of encapsulated protein but also showed incomplete release of protein from nanoparticle over a period of $40 \mathrm{hrs}$. Ionotropic gelation technique was a suitable technique for preparing chitosan-TPP nanoparticles with a nano-size range and also successfully protein loaded. The amount of chitosan and BSA had great effect on the protein loading, encapsulation efficiency, size and zeta potential. For the inorganic nanocarriers, the CTAB based silica nanoparticles were porous before protein loading and this porous structure enabled successful loading of protein. Silica nanoparticles prepared from $0.8 \%$ CTAB with $2 \mathrm{ml}$ TEOS solution was considered optimised formulation according to protein release profile, morphology and nanoparticles stability and was considered for further study. Finally, the optimised PLGA, chitosan and silica nanoparticles exhibited similar sustained BSA release profiles and the rate of release from all three type of nanoparticles was not significantly different, suggesting, that factors such as the properties of the protein and the dissolution may play an important role in drug release from these matrices.

\section{Future Perspective:}

Though very few nanoparticle based formulations have reached the market, the interest in these versatile formulations will continue to increase, driven mainly by the advances in biotechnology and new biomacromolecules such as peptides, proteins, nucleotides, and even stem cells, that require effective protection afforded by encapsulation within nanoparticulate formulations, due to their poor stability. 
Furthermore, the need for targeting such biomacromolecules to specific sites of action, including cancer, vaccine delivery as well as avoiding destructive enzymes, will continue to drive an interest in nanoparticles. This is because nanocarriers have the ability to allow surface functionalisation, which enhances controlled release in time and site specific ways. Finally, the need to avoid painful injections, especially in the paediatric and geriatric populations will drive interest in alternative formulations to allow easy oral administration, as is the case for oral mucosal vaccination, which will also avoid first pass metabolism and therefore allow lower administered doses, to boost safety profiles.

\section{Executive Summary}

\section{Background}

- Nano carriers can help to deliver therapeutic proteins via the oral route. This study aimed to develop compare nanocarriers obtained from three different types of materials using BSA as a model protein antigen.

- This is the first study that systematically compares nanoparticles obtained from synthetic organic (PLGA), natural organic (chitosan), and inorganic starting materials and simultaneously investigate the effect of process and formulation variables on the performance characteristics of the resulting nanoparticles for potential protein delivery.

\section{Methods}

- PLGA nanoparticles were prepared by solvent emulsification evaporation and double emulsion, chitosan nanoparticles using inotropic gelation and silica nanoparticles by sol-gel synthesis process. Finally, BSA was loaded into selected optimised PLGA, chitosan and silica nanoparticles.

- The nanoparticles were characterised for size, zeta potential and PDI using DLS including the effect of formulation and process parameters, while protein loading, encapsulation and release were determined using Bradford assay. Physical properties of starting materials and formulated nanoparticles were characterised using SEM, TEM, STEM, XRD, FTIR and in vitro drug release for BSA loaded formulations. Finally, the functional properties of the 3 different nanoparticles (PLGA, chitosan and silica) were compared.

\section{Effect of concentration of polymer, silica and surfactant on particle properties.}

- The amount of surfactant and polymer affected the particle size, PDI and zeta potential of PLGA nanoparticles. For chitosan nanoparticles, the particle size, PDI and zeta potential increased with increasing chitosan concentration. Monodisperse particles with uniform size was 
observed for the silica nanoparticles prepared with $\mathrm{CTAB}$ concentration of $0.9 \%$ and was chosen as optimum concentration for protein loading.

\section{Effect of homogenization}

- Faster stirring produced more stable PLGA particles based on the zeta potential. For a fixed stirring speed, the particle size decreased and zeta potential increased with increasing homogenization time.

- For chitosan nanoparticles, increasing homogenization speed from 1000 to $7000 \mathrm{rpm}$ reduced size from 325 to $277 \mathrm{~nm}$ while effect of homogenisation time was inconclusive.

- The size of the silica nanoparticles decreased from 246 to $185 \mathrm{~nm}$ with increasing homogenizer speeds, however, increasing homogenization time did not significantly affect the particle size. However, PDI decreased and zeta potential increased with homogenisation, which increased stability and monodispersed nature of the nanoparticle suspension.

- Generally, the effect of homogenization appeared to be more pronounced on size and PDI of PLGA nanoparticles compared to the chitosan and silica nanoparticles whilst the differences in effect on zeta potential between the three types of formulations was less pronounced.

\section{Effect of ultra-sonication}

- Within 2 min of probe sonication, PLGA particles became monodispersed and PDI decreased subsequently optimum sonication time determined at $4 \mathrm{~min}$. For chitosan nanoparticles average diameter of chitosan nanoparticles decreased after sonication, but the particle size reduction rate after $8 \mathrm{~min}$ of sonication was not significant. Zeta potential also increased while PDI values decreased between 1 and 4 min of sonication time. With increased ultra-sonication time silica particles size decreased which reduced the extent of agglomeration.

- Unlike homogenization, where PLGA nanoparticles were most affected, probe sonication affected the silica nanoparticles more compared to the optimised PLGA and chitosan nanoparticles. The silica nanoparticles showed significant reduction in particle size below 200nm after 6 minutes of sonication, whilst the PLGA and chitosan nanoparticles remained far higher than $200 \mathrm{~nm}$ even after $12 \mathrm{~min}$ of probe sonication.

\section{BSA loading efficiency of nanoparticles}

- All the optimised nanoparticles fell within the preferred size range for protein based vaccine delivery which is 200 to $500 \mathrm{~nm}$.

- Blank PLGA-PVA nanoparticle which had an original size of $244 \mathrm{~nm}$, increased in size to 292 $\mathrm{nm}$ after protein loading. For chitosan nanoparticles, changing chitosan concentrations $(0.1 \%$ to $0.4 \%$ ) increased EE from $68 \%$ to $72 \%$, and LC from 27 to $48 \%$. After protein loading, the 
particles size also increased from 221 to $246 \mathrm{~nm}$. All silica nanoparticles had a negative surface charge and zeta potential increased and remained negative after BSA loading and size also increased from 176 to $201 \mathrm{~nm}$.

- PLGA and silica formulations had negative zeta potential whilst chitosan remained positive. Silica nanoparticles showed higher zeta potential values than the PLGA and chitosan suggesting the inorganic particles were more physically stable than the organic equivalents.

\section{Morphology of the nanoparticles by electron microscopy}

- PLGA nanoparticles were of spherical shape with size range between $200 \mathrm{~nm}$ to $1 \mu \mathrm{m}$ Chitosan $0.1 \%$ nanoparticles showed a high number of particles formed but with varied sizes ranging between $200 \mathrm{~nm}$ to $1 \mu \mathrm{m}$. Optimised silica 9 , showed smooth surface and size range between $200 \mathrm{~nm}$ to $1.5 \mu \mathrm{m}$.

- TEM and STEM confirmed the encapsulation of BSA within the optimised PLGA, chitosan and silica nanoparticles.

\section{In vitro protein release study}

- The nanoparticles were prepared from three different starting materials (synthetic organic, natural organic and inorganic), all showed similar sustained release profiles.

- This suggests that the properties of the drug and the medium environment, play an important role in determining drug release characteristics from nanoparticles prepared from different materials.

\section{REFERENCES}

[1] Lombardo D, Kiselev MA, Caccamo MT. Smart nanoparticles for drug delivery application: Development of versatile nanocarrier platforms in biotechnology and nanomedicine. J. Nanomater. 2019, 1-26 (2019). * This article explores recent advances in stimuli responsive nanocarriers prepared using organic and inorganic materials, for efficient delivery of drugs in the harsh biological environment within the body, including diseased organs and tissues.

[2] Shukla SK, Sudheesh SK, Govender PP, Giri NG. Biodegradable polymeric nano-structures in therapeutic applications: opportunities and challenges. RSC Adv, 6, 94325-94351 (2016).

[3] Talelli M, Duro-Castano A, Rodríguez-Escalona G, Vicent MJ. Smart polymer nanocarriers for drug delivery. Smart Polym. Applic. 11, 327-358 (2014).

[4] Sharma R, Agrawal U, Mody N, Vyas SP. Polymer nanotechnology based approaches in mucosal vaccine delivery: Challenges and opportunities', Biotechnol. Adv. 33(1), 64-79 (2015).

[5] Liechty WB, Kryscio DR, Slaughter BV, Peppas NA. Polymers for Drug Delivery Systems. 
Annual. Rev. Chem. Biomol. Eng. 1, 149-173 (2010).

[6] Gregory AE, Titball R, Williamson D. Vaccine delivery using nanoparticles. Front. Cell. Infect. Microbiol. 3, 1-13 (2013). * This is a review article that compares the use of different nanoparticulate carriers for vaccine delivery, which is one of the key objectives in our study and confirms the potential of nanocarriers to overcome some of the challenges with current vaccine administration.

[7] Kreuter J. Nanoparticles and microparticles for drug and vaccine delivery. J. Anat. 189(3), 503-505 (1996).

[8] Feczkó T, Tóth J, Dósa G, Gyenis J. Optimization of protein encapsulation in PLGA nanoparticles. Chem. Eng. Process. Proc Intensif. 50(8), 757-765 (2011).

[9] Morales-Cruz M, Flores-Fernández GM, Orellano EA, Rodriguez-Martinez JA, Ruiz M, Griebenow K. Two-step nanoprecipitation for the production of protein-loaded PLGA nanospheres. Results Pharm. Sci. 2, 79-85 (2012).

[10] Mishra N, Tiwari S, Vaidya B, Agrawal GP, Vyas SP. Lectin anchored PLGA nanoparticles for oral mucosal immunization against hepatitis B. J. Drug Target. 19(1), 67-78 (2011). * This paper confirms the potential of PLGA based nanoparticles as potential drug delivery carrier for antigen delivery targetting peyer's patches for mucosal vaccination and further confirms the importance of ensuring optimised physico-chemical properties such as size, shape and physical stability.

[11] Mao S, Sun W, Kissel T. Chitosan-based formulations for delivery of DNA and siRNA. Adv. Drug Del. Rev. 62(1), 12-27 (2010).

[12] Amidi M, Mastrobattista E, Jiskoot W, Hennink WE. Chitosan-based delivery systems for protein therapeutics and antigens. Adv. Drug Del. Rev. 62(1), 59-82 (2010).

[13] Lin YH, Mi FL, Chen CT et al. Preparation and characterization of nanoparticles shelled with chitosan for oral insulin delivery. Biomacromol. 8(1), 146-152 (2007). * This shows the ability of chitosan to protect insulin, a labile biomacromolecule for oral delivery, thus potentially avoiding the need for injections.

[14] Jarudilokkul S, Tongthammachat A, Boonamnuayvittaya V. Preparation of chitosan nanoparticles for encapsulation and release of protein. Korean J. Chem. Eng. 28, 1247-1251 (2011).

[15] Van der Lubben IM, Kersten G, Fretz MM, Beuvery C, Coos Verhoef J, Junginger HE. Chitosan microparticles for mucosal vaccination against diphtheria: Oral and nasal efficacy studies in mice. Vaccine, 21(13-14), 1400-1408 (2003).

[16] Tiyaboonchai W, Chitosan Nanoparticles : A Promising System for Drug Delivery. Naresuan Univ. J. 11(3), 51-66 (2003).

[17] Tang L, and Cheng J. Nonporous silica nanoparticles for nanomedicine application," Nano Today, 8(3), 290-312 (2013). 
[18] Mody KT, Popat A, Mahony D, Cavallaro AS, Yu C, Mitter N. Mesoporous silica nanoparticles as antigen carriers and adjuvants for vaccine delivery. Nanoscale. 5(12), 51675179 (2013). * This article provides an overview of the potential of mesoporous (inorganic) silica nanoparticles for delivering antigens to elicit immune responses and highlights the importance of judicious formulation and selection of appropriate antigen for eliciting the required immune response.

[19] Li L, Liu T, Fu C, Tan L, Meng X, Liu H. Biodistribution, excretion, and toxicity of mesoporous silica nanoparticles after oral administration depend on their shape. Nanomed. Nanotechnol. Biol. Med. 11(8), 1915-1924 (2015). ** In our study, we highlighted the importance of optimising the basic formulation and process parameters that impact nanoparticle performance and this study confirms the important effects of functional physicochemical properties such as the shape on the biopharmaceutics performance of silica based nanoparticles.

[20] Wang T, Jiang H, Zhao Q, Wang S, Zou M, Cheng G. Enhanced mucosal and systemic immune responses obtained by porous silica nanoparticles used as an oral vaccine adjuvant: Effect of silica architecture on immunological properties. Int. J. Pharm. 436(1-2), 351-358 (2012). * This study investigated the relationship between silica morphological architecture, its physical characteristics such as size, porosity and the resulting immunological response when used as oral muosa vaccine delivery system and is an important addition to the field.

[21] Sharma N, Madan P, Lin S. Effect of process and formulation variables on the preparation of parenteral paclitaxel-loaded biodegradable polymeric nanoparticles: A co-surfactant study. Asian J. Pharm. Sci. 11(3), 404-416 (2016).

[22] Fan W, Yan W, Xu Z, Ni H. Formation mechanism of monodisperse, low molecular weight chitosan nanoparticles by ionic gelation technique. Coll. Surf. B: Biointerf. 90, 21-27 (2012).

[23] Jafarzadeh M, Rahman IA, Sipaut CS. Synthesis of silica nanoparticles by modified sol-gel process: The effect of mixing modes of the reactants and drying techniques. J. Sol-Gel Sci. Technol. 50(3), 328-336 (2009). * This article confirms the importance of paying attention to critical process parameters in the formulation of silica nanoparticles to ensure optimised physico-chemical properties to assure effective drug delivery.

[24] Wang JJ, Zeng ZW, Xiao RZ et al. Recent advances of chitosan nanoparticles as drug carriers. Int. J. Nanomedicine. 6, 765-774 (2011).

[25] Qinna NA, Karwi QG, Al-Jbour N et al. Influence of molecular weight and degree of deacetylation of low molecular weight chitosan on the bioactivity of oral insulin preparations. Mar. Drugs. 13(4), 1710-1725 (2015).

[26] Bhumkar DR, Pokharkar VB. Studies on effect of $\mathrm{pH}$ on cross-linking of chitosan with sodium tripolyphosphate: A technical note. AAPS PharmSciTech. 7(2), 138-143 (2006).

[27] Barbé C, Bartlett J, Kong L et al. Silica particles: A novel drug-delivery system. Adv. Mater. 
16(21), 1959-1966 (2004).

[28] Derman S, Mustafaeva ZA, Abamor ES, Bagirova M, Allahverdiyev A. Preparation, characterization and immunological evaluation: canine parvovirus synthetic peptide loaded PLGA nanoparticles. J. Biomed. Sci. 22, 89-101 (2015).

[29] Tang BC, Dawson M, Lai SK et al. Biodegradable polymer nanoparticles that rapidly penetrate the human mucus barrier. Proc. Natl. Acad. Sci. 106(46), 19268 -19273 (2009).

[30] Raj LFAA, Jonisha R, Revathi B, Jayalakshmy E. Preparation and characterization of BSA and chitosan nanopartices for sustainable delivery system for quercetin. J. Appl. Pharm. Sci. 5(7), 1-5 (2015).

[31] Gan Q, Wang T. Chitosan nanoparticle as protein delivery carrier-Systematic examination of fabrication conditions for efficient loading and release. Coll. Surf. B: Biointerf. 59(1), 24-34 (2007).

[32] Han Y, Duan Q, Li Y, Li Y, Tian J. Preparation and Characterization of Chitosan-Based Nanoparticles as Protein Delivery System. Adv. Polym. Technol. 37(4), 1214-1220 (2018).

[33] Sarmento B, Ribeiro A,Veiga F, Ferreira D. Development and characterization of new insulin containing polysaccharide nanoparticles. Coll. Surf. B: Biointerf. 53(2), 193-202 (2006).

[34] Liao YT, Wu KC, Yu J. Synthesis of mesoporous silica nanoparticle-encapsulated alginate microparticles for sustained release and targeting therapy. J. Biomed. Mater. Res. - Part B Appl. Biomater. 102(2), 293-302 (2014).

[35] Shakeri S, Roghanian R, Emtiazi G, Errico C, Chiellini F, Chiellini E. Preparation of proteinloaded PLGA-PVP blend nanoparticles by nanoprecipitation method: entrapment, Initial burst and drug release kinetic studies. Nanomed. J. 2(3), 175-186 (2015).

[36] Budhian A, Siegel SJ, Winey KI. Production of haloperidol-loaded PLGA nanoparticles for extended controlled drug release of haloperidol. J. Microencapsul. 22(7), 773-785 (2005).

[37] Hamishehkar H, Emami J, Najafabadi AR et al. The effect of formulation variables on the characteristics of insulin-loaded poly(lactic-co-glycolic acid) microspheres prepared by a single phase oil in oil solvent evaporation method. Coll. Surf. B: Biointerf. 74(1), 340-349 (2009).

[38] Manchanda R, Fernandez-Fernandez A, Nagesetti A, McGoron AJ. Preparation and characterization of a polymeric (PLGA) nanoparticulate drug delivery system with simultaneous incorporation of chemotherapeutic and thermo-optical agents. Coll. Surf. B: Biointerf. 75(1), 260-270 (2010).

[39] Mittal G, Sahana DK, Bhardwaj V, Ravi Kumar MN. Estradiol loaded PLGA nanoparticles for oral administration: Effect of polymer molecular weight and copolymer composition on release behavior in vitro and in vivo. J. Control. Rel. 119(1), 77-85 (2007). 